STEPHAN WOLFF

\title{
Gepflegte Intransparenz \\ Zur Funktion von Ironie in der Kommunikation vor Gericht
}

\section{Ernst und Verfahren}

Obwohl das räumliche Arrangement, die sorgfältig kalkulierte Dramaturgie der Verhandlungsschritte und gelegentlich auch die bei Gericht verhandelten Geschichten bisweilen an Theateraufführungen erinnern, und obwohl gerade der Strafprozess und seine Beteiligten beliebte Anlässe und Zielscheiben für die gesellschaftliche Witzproduktion abgeben, erweisen sich Gerichtsverfahren im Großen und Ganzen als eine vergleichsweise ernste Angelegenheit.

Ein wesentlicher Grund für die sprichwörtliche Ernsthaftigkeit von Gerichtsverfahren und insbesondere von Strafprozessen liegt sicherlich darin, dass vor Gericht an die Beteiligten hohe Anforderungen bezüglich der Konsistenz ihrer (Selbst-)Darstellungen gestellt werden. Niklas Luhmann bringt dies mit der Ausrichtung von Verfahren auf allgemein verbindliche Entscheidungen in Verbindung. ${ }^{1}$ Im Hinblick darauf muss sicher gestellt sein, dass an die beabsichtigten wie an die unbeabsichtigten Informationen, welche die Beteiligten im Verlaufe der Verhandlung geben, zuverlässig angeknüpft werden kann.

„Alle Beteiligten werden durch den Sinn und das Zeremoniell des Verfahrens angehalten, ihr Verhalten Ernst zu nehmen und es als bindend zu betrachten wie eine Kette von Versprechungen, und sie tun gut, diesen Erwartungen durch Vorprüfung ihres Verhaltens Rechnung zu tragen."

Von den professionellen Akteuren - von den beteiligen Juristen, den diversen Gutachtern und dem sonstigen Hilfspersonal - wie von den im Gerichtssaal nur gelegentlich auftretenden ,Laienspielern', - d. h. von den Angeklagten und Zeugen -, wird ein gewisses Maß an nüchterner Unpersönlichkeit erwartet. Institutionelle Verfahren sind gera-

\footnotetext{
1 Siehe NIKLAS LuHManN, Legitimation durch Verfahren. Neuwied 1969.

2 LUHMANN, Legitimation durch Verfahren (Fn. 1), 92.
} 
dezu darauf angelegt, ,Persönlichkeit' einzufangen und zu binden. Dies geschieht insbesondere dadurch, dass bestimmte, im alltäglichen Verkehr frei zugängliche kommunikative Instrumentarien für die Gestaltung sozialer Situationen während der Verhandlung nur eingeschränkt verfügbar sind.

"Obgleich man Ansätze zur und Bemühungen um Distanzierung von der eigenen Rolle bei Prozessparteien und vor allem natürlich bei Angeklagten im Strafprozess immer wieder beobachten kann, werden ihnen durch das Zeremoniell und die offizielle Ernsthaftigkeit des Geschehens die wichtigsten Ausdrucksmittel wie Scherz, Übertreibung, Lässigkeit usw. genommen. ${ }^{33}$

Eine wichtige Leistung institutioneller Verfahren besteht darin, dass dort offiziell jeder meint, was er sagt, und sagt, was er meint. ,Ernste' Kommunikation wird durch gesellschaftliche Institutionen überhaupt erst möglich - und umgekehrt. Die Übereinstimmung von Sagen und Meinen ist vorreguliert und diese Vorregulierung ihrerseits wird ernst genommen und gegebenenfalls sanktioniert. Wenn also Dirk Baecker davon spricht, dass Witz, Ironie und Albernheit bei dieser Form der Kommunikation nicht dazu gehören, dort keinen Eindruck machen, dort nichts ändern und einem im Halse stecken bleiben, ${ }^{4}$ so hat der mit dieser These sicherlich recht - wenn man den Blick lediglich auf die offizielle Darstellung und auf die Programmatik des Verfahrens richtet.

Betrachtet man die tatsächlich bei Gericht ablaufende Kommunikation jedoch genauer, dann erweist sich diese Feststellung als ein wenig $\mathrm{zu}$ apodiktisch formuliert. Sicherlich, auch nach unseren Beobachtungen finden sich im Strafverfahren humorige Handlungsweisen wie Scherzen, Witze machen und Frotzeln ziemlich selten und werden, sollten sie überhaupt auftauchen, von den Betreffenden in aller Regel als von der offiziellen Linie abweichende Verhaltensweisen eigens markiert. So kommentierte ein von uns beobachteter Richter einen Scherz mit den Worten: „Wenn Sie mir die Bemerkung gestatten“.

Vergleichweise häufig und dies gerade, wenn es im Verfahren ,ernst' wird, kommen dagegen Ironisierungen vor. Ironische Bemerkungen betreffen fast ausschließlich Aussagen bzw. Eigenschaften von Angeklagten und Zeugen. Die primäre Zielscheibe entsprechender Bemerkungen scheint der Wahrheitsgehalt solcher Aussagen und damit verbunden die Glaubwürdigkeit ihrer Urheber zu sein. ${ }^{5}$

\footnotetext{
${ }^{3}$ LUHMANN, Legitimation durch Verfahren (Fn. 1), $98 \mathrm{f}$.

${ }^{4}$ Siehe DIRK BAECKER, Ernste Kommunikation, in: KARL HEINZ BOHRER (Hrsg.), Sprachen der Ironie, Sprachen des Ernstes. Frankfurt am Main 2000, 389-403.

${ }^{5}$ Dieser Aufsatz schließt an zwei Arbeiten des Verfassers zusammen mit Hermann Müller (STEPHAN WOLFF/HERMANN MÜLLER, Ironie als Instrument der Wahrheitsfindung", in: Zeitschrift für Soziologie 24 (1995), 451-464; STEPHAN WOLFF/IERMANN MULLER, Kompetente Skepsis. Eine konversationsanalytische Untersuchung zur Glaubwiirdig-
} 


\section{Definitorische Annäherungen an Ironie}

Wir nähern uns dem Phänomen der Ironie zunächst auf dem üblichen Weg, indem wir nämlich nach Abgrenzungskriterien suchen und zu diesem Zweck auf die etablierte Literatur zurückgreifen.

Das griechische Eironeia, aus dem sich unser Wort Ironie herleitet, bezeichnet im planen Sinn die Verstellung, sei es in Form der Überoder der Untertreibung. Insbesondere in der Rhetorik blickt die Ironie auf eine lange Tradition zurück. Schon bei Cicero fungiert die Figur der Ironie als Ausdrucksmittel und Instrument der forensischen Beredsamkeit. Gemäß Quintilian meint die Ironie das Gegenteil von dem, was mit den Worten gesagt wird. Zu Beginn des 19. Jahrhunderts taucht mit der sog. "romantischen" eine neue Form der Ironie auf. Friedrich Schlegel bezeichnet mit seinem Ironie-Begriff nicht mehr die nunmehr als trivial empfundene rhetorische Figur, sondern ein "philosophisches Vermögen", und eine Philosophie, die sich der Grenzen ihrer Sagbarkeit bewusst wird. Er kennzeichnet deren Funktionsweise in den Athenäums-Fragmenten wie folgt:

"Die romantische Ironie kann am meisten zwischen dem Dargestellten und dem Darstellenden, frei von allem realem und idealem Interesse auf den Flügeln der poetischen Reflexion in der Mitte schweben, diese Reflexion immer wieder potenzieren und wie in einer Reihe von Spiegelungen vervielfachen."6

Theodor Lewandowskis Linguistisches Wörterbuch bestimmt Ironie ein wenig prosaischer als

„eine Form des uneigentlichen und bewertenden Sprechens, bei der ein Spannungsverhältnis zwischen der wörtlichen Bedeutung der Äußerung und der mit der Äußerung intendierten Bedeutung besteht". ${ }^{7}$

Wir lernen also: Ironie sagt zwar nicht notwendig das Gegenteil vom dem, was sie meint, aber immer etwas anderes. ${ }^{8}$ Meinen und Sagen fallen auch aus der Sicht der Beteiligten erkennbar auseinander. Der $D u$ den bietet in dieser Hinsicht eine nicht unwesentliche Präzisierung: Er versteht unter Ironie einen

"feinen verdeckten Spott mit dem man etwas dadurch zu treffen versucht, dass man es unter dem augenfälligen Schein der Billigung lächerlich macht."

keit im Strafuerfahren. Opladen 1997) an, die im Kontext eines von der Stiftung Volkswagenwerk geförderten Projekts zur Glaubwürdigkeit in Strafverfahren entstanden sind Für die institutionelle Betreuung sei noch einmal Professor Hagen Hof herzlich gedankt.

${ }^{6}$ Athenaeum: Eine Zeitschrift. Herausgeben von AUGUST WILHELM SCHLEGEL und FRIEDRICH SCHLEGEL. 3 Bde. Berlin 1798-1800. Bd. 1, 93, 95.

${ }^{7}$ THEODOR LEWANDOWSKI, Linguistisches Wörterbuch. 5. Aufl., Heidelberg 1990, 497 f

8 UWE JAPP, Theorie der Ironie. Frankfurt am Main 1983. 
Daraus folgt zum einen, dass es ein Objekt der Ironie gibt, das der Ironiker zu treffen versucht. Zum anderen, dass es sich bei der gelingenden Ironie um eine widersprüchliche Beziehung von , augenfälliger Verdecktheit' und ,scheinhafter Billigung' handelt. Beides, Verdecktheit und Billigung, werden zusammen mit ihrer Negation kommuniziert, und zwar ohne dass es zu einem performativen Widerspruch kommen würde. Noch eine dritte Implikation gilt es zu notieren: Es kann beim bloßen ,Versuch' der Ironie bleiben. Ironisierungen scheitern, wenn eine derartige Balance in der kommunikativen Situation nicht gelingt. Die Kunst des Ironikers bzw. der an einer Ironisierung Beteiligten scheint eben darin zu bestehen eine solche widersprüchliche Konstellation aufrechtzuerhalten.

Bollnow beschreibt die Leistung der Ironie noch ein wenig genauer, indem er feststellt, das für die Ironie Charakteristische sei das „in der Schwebe halten" des kommunikativen Geschehens zwischen Ernstnehmen und Nicht-Ernstnehmen:

„Die in der Ironie beabsichtigte Wirkung ist ... die, dass der Betroffene sie weder schlechthin durchschaut noch schlechthin auf sie hereinfällt, sondern stutzig wird - möglichst erst nachträglich, nachdem er sie zuvor für bare Münze genommen hat - und dann im Zweifel zwischen den beiden Möglichkeiten hin- und her geworfen wird. Erst hier kommt ein für die Ironie entscheidender Zug ans Licht: dass man nicht weiß, wie weit man das ironische Verhalten ernst nehmen soll und wie weit nicht und so gerade durch diese Unsicherheit in der Schwebe gehalten wird."

Wer sich, wie wir, dem Phänomen der Ironie empirisch nähern will, der kann bei solchen definitorischen Abgrenzungen freilich nicht stehen bleiben, sondern muss sich mit dem ,Wie', d. h. mit der systematischen Rekonstruktion ihrer interaktiven Realisierung an empirischem Material beschäftigen ${ }^{10}$. Wir lesen derartige Definitionen deshalb lediglich als heuristische Hinweise auf strukturelle Handlungsanforderungen, welche sich den Gesprächsteilnehmern bei der interaktiven Realisierung von Ironie nach Meinung dieser Autoren stellen.

Alle angeführten Definitionen waren sich einig, dass der Ironisierende mit seiner Äußerung etwas feststellt, was er eigentlich so nicht meint. Ein wesentliches strukturelles Handlungsproblem scheint mithin darin zu bestehen, in der Aussage neben dem offiziellen Text noch eine Gegenposition nicht nur mitschwingen zu lassen, sondern diese sogar zur wesentlichen Botschaft zu machen. Der Rezipient einer ironi-

\footnotetext{
${ }^{9}$ OTTO F. BOLLnOW, Ironie, in: DERS., Die Ehrfurcht. 2. Aufl., Frankfurt am Main 1958, 147 ff., $152 \mathrm{f}$.

${ }^{10} \mathrm{Vgl}$. als klassische Formulierung des konversationsanalytischen Vorgehens HARVEY SACKS, Lectures on Conversation. 2 Bände, Oxford 1992.
} 
schen Äußerung muss in der Lage sein bzw. in die Lage versetzt werden, zwei sich widersprechende Botschaften wahrzunehmen, um die Äußerung als eine ironische erkennen, und darüber hinaus die ,wirkliche Bedeutung' des Gesagten herausfinden zu können.

Dies ist keineswegs trivial: ironische Bemerkungen gehen bekanntlich leicht daneben. Bei der Ironie als einer Form "uneigentlichen Sprechens $^{\prime \prime 11}$ bleiben die Signale, die den Hörer oder Leser darauf hinweisen, dass eine Äußerung nicht wörtlich, sondern anders zu verstehen ist, immer einigermaßen vage und unsicher. Diese Vagheit und partielle Intransparenz scheinen andererseits aber konstitutiv für Ironie zu sein. Allzu klare Interpretationsanweisungen an die Hörer würden nämlich den ironischen Charakter der Äußerung zerstören oder deren Wirkung schmälern. Für das Glücken einer Ironie scheint eine angemessene Reaktion der Rezipienten eine notwendige Bedingung zu sein. Angesichts dessen muss in der Gerichtskommunikation die interpretative wie die interaktive ,Mitarbeit' der Rezipienten, $d$. h. auch und gerade der vermeintlichen, Opfer' ironisch (gemeinter) Äußerungen sichergestellt werden.

Damit sehen wir uns mit einem eigenartigen Tatbestand konfrontiert: In einem programmatisch derart auf Eindeutigkeit und Ernsthaftigkeit ausgerichteten Interaktionssystem wie dem Gerichtsverfahren begegnen wir mit ironischen Äußerungen einem Phänomen,

- das anscheinend erst dann seine soziale Wirkung entfaltet, wenn man es kaum noch wahrnimmt;

- das es den Akteuren unmöglicht macht, das, was gemeint ist, ausdrücklich anzusprechen, wollen sie nicht die beabsichtigte Wirkung gefährden;

- das nur dann glücken kann, wenn auch die dadurch benachteiligte Partei mitspielt.

Um uns Aufklärung über die Struktur, Dynamik und Funktion dieses eigenartigen Phänomens zu verschaffen, bleibt nichts anderes übrig als sich Ironie als praktisches Interaktionshandeln vor Ort, $d$. h. seine spezifische Verwendung im Ablauf realer Gerichtsverhandlungen genauer anzusehen. Dabei lassen wir uns von der heuristischen Unterstellung leiten, dass die offensichtliche Intransparenz der Ironie ,Methode hat', und betrachten Ironie demzufolge als eine kunstvolle Leistung der beteiligten Akteure. Die für einen Konversationsanalytiker interessanten Fragestellungen lautet somit: Was macht die Methodizität ironi-

11 WOLFGANG BERG, Uneigentliches Sprechen. Zur Pragmatik und Semantik von Metapher, Metonymie, Ironie und rhetorischer Frage. Tübingen 1978. 
schen Sprechens aus, welcher kommunikativer Instrumentarien bedienen sich die Beteiligten dabei und welche Funktion kommt derartigen „un-ernsten“ Episoden im Gerichtsverfahren zu?

Hierzu sind zunächst die für Ironie typische Beteiligtenkonstellation sowie die kommunikative Struktur einer ironischen Episode („Ironiesequenz") zu klären. Dies sollte uns dann in die Lage versetzen genauer zu bestimmen, welche besonderen interaktiven Funktionen ironische Passagen im Strafverfahren erfüllen, bzw. wie sie von den verschiedenen Beteiligten in strategischer Absicht eingesetzt werden können.

\section{Die Beziehungsstruktur der Ironie}

Als Produzenten ironischer Äußerungen kommen in Strafverfahren primär die juristischen Hauptakteure in Frage. In dem uns vorliegenden Material ${ }^{12}$ stellen von der Zahl der Fälle her die Richter eindeutig die Hauptgruppe der Ironisierer, gefolgt von den Staatsanwälten und mit weitem Abstand - den Verteidigern. Dieses Übergewicht der Richter relativiert sich, wenn man berücksichtigt, dass sie im deutschen Strafverfahren etwa $80 \%$ der Befragungen bestreiten. Ironische Bemerkungen richten sich auf Äußerungen von Angeklagten und Zeugen; sie sind somit die primären Objekte der Ironisierung. ${ }^{13}$ Wir haben keine Beispiele dafür gefunden, in denen Sachverständige oder gar Richter ironisiert werden. Dabei fällt auf, dass Äußerungen ,neutraler Zeugen' und von vornherein geständiger Angeklagter so gut wie nie ironisiert werden. Auf der anderen Seite finden sich aber auch keine Beispiele, in denen dieser Personenkreis als Subjekt der Ironisierung auftreten würde.

\footnotetext{
12 Dieses Datenmaterial besteht aus Tonbandaufzeichnungen von 36 Verfahren, von denen 22 als Transkriptionen vorliegen. Der Anklagevorwurf bezog sich überwiegend auf kleinere und mittelschwere Straftaten. Hinzu kommen Verfahrenbeobachtungen Dokumentenanalysen von Urteilsgründen und Gutachten sowie Expertengespräche. $\mathrm{Zu}$ Details des Untersuchungsdesigns vgl. WOLFF/MÜLLER, Kompetente Skepsis (Fn. 5), $32 \mathrm{ff}$.

${ }^{13} \mathrm{Zu}$ finden sind darüber hinaus auch ironische Seitenhiebe in den Plädoyers der Staatsanwälte und Verteidiger, beispielsweise, wenn sie sich auf Art und Stringenz der Ausführungen der jeweils anderen Seite beziehen. Dies stellt einen besonderen Verwendungszusammenhang der Ironie im Verfahren dar, den wir im Folgenden unberücksichtigt lassen. Ironisierungen dienen hier der kommunikativen Austragung von Meinungsverschiedenheiten in einer Weise, dass dadurch massive Kritik geäußert werden kann, ohne aber dass das Gegenüber als Professioneller oder gar die juristische Profession als solche zu gefährden. Analoge Formen und Funktionen von ironischen Bemerkungen lassen sich in sog. „Obergutachten“ beobachten, in denen Gutachter über Gutachter urteilen, STEPHAN WOLFF, Text und Schuld. Die Rhetorik psychiatrischer Gerichtsgutachten. Berlin 1995.
} 
Dass wir solche Fälle nicht gefunden haben, heißt freilich nicht, dass sie nicht vorkommen könnten. Die Reaktionen der Beteiligten auf solche, seltenen ,abweichenden Fälle' demonstrieren aber ihrerseits die besondere Interaktionsordnung vor Gericht. ${ }^{14}$ Fälle der Ironisierung durch Zeugen oder Angeklagte, insbesondere Ironisierungen von Richtern, werden durchweg als Übergriffe empfunden und führen regelmäßig zu Verfahrenskrisen. Ihre interaktionsstrukturell fundierte Unwahrscheinlichkeit lässt solche Vorkommnisse $\mathrm{zu}$ außerordentlich bemerkenswerten Ereignissen werden. Manche dieser Übergriffe erlangen über die Gerichtsszene hinaus an Berühmtheit. Jedem heutigen Juristen dürfte beispielsweise Fritz Teufels Bemerkung "Wenn's der Wahrheitsfindung dient!" geläufig sein, mit der dieser 1967 die Aufforderung eines Richters „Erheben Sie sich zu Ehren des Gerichtes, Angeklagter!" quittierte.

Erwähnt werden muss noch eine dritte, wenngleich nicht obligatorische Teilnehmerkategorie: das Ironiepublikum. ${ }^{15}$ Die Dramaturgie des Gerichtsverfahrens verweist auf ein engeres und ein weiteres Publikum, wobei in aller Regel nur das engere ausdrücklich adressiert wird. Darüber hinausgehende, d. h. das Publikum auf den Zuschauerbänken einbeziehende oder auf eben dieses zielende Adressierungen werden von den Richtern, aber auch von den anderen Beteiligten als ,Grenzüberschreitungen' markiert und gegebenenfalls mit Ordnungsrufen oder Befangenheitsanträgen sanktioniert. Eine weitere Besonderheit ironischer Episoden vor Gericht besteht darin, dass das Publikum zwar anwesend ist, in aller Regel aber keine offenen Reaktionen (wie Lachen) zu beobachten sind. Auch die Ironisierer selbst halten sich diesbezüglich zurück, zumindest soweit dies unseren Tonbandaufzeichnungen und Transkripten zu entnehmen ist.

\footnotetext{
${ }^{14}$ In der Konversationsanalyse ist deshalb die so genannte "deviant case analysis" ein außerordentlich wichtiges Validierungsverfahren.

15 Weinrich spricht deshalb von einem "triadischen Ironiemodell”, räımt aber ein: „Es mag sein, dass kein Dritter da ist"; vgl. HARALD WEINRICH, Ironie, in: JOACHIM RITTER/ KARLFRIED GRÜNDER (Hrsg.), Historisches Wörterbuch der Philosophie. Bd. 4. Darmstadt 1976, 577-582. Diese Bemerkung bezieht sich auf das Vorkommen von Ironie in ZweipersonenGesprächen, in denen die Position des Zuhörers wegfällt bzw. vom Sprecher und vom Adressaten - auf empirisch noch kaum geklärte Weise - mit erledigt werden muss.
} 


\section{Strukturmerkmale ironischen Sprechens}

\subsection{Orte der Ironie in der Vernehmung}

Der Hang zu ironischen Bemerkungen hat zweifellos etwas mit dem jeweiligen persönlichen Stil zu tun. Allerdings kann sich persönlicher Stil letztlich immer nur situationsbezogen mit Rücksicht auf den sequentiellen, thematischen, lexikalischen und kategorialen Kontext entfalten. Ironisierungen kommen dementsprechend keineswegs aus heiterem Himmel. Wir finden sie bevorzugt an bestimmten Orten im Verfahrensablauf platziert. Nach unseren Beobachtungen werden ÄuBerungen von Zeugen oder Angeklagten nur dann ironisiert, wenn bereits vorher Hinweise auf eine mögliche Unglaubwürdigkeit dieser Personen interaktiv herausgearbeitet wurden. Wir wollen uns deshalb zuerst einmal den größeren strukturellen Zusammenhang ansehen, in dem Ironisierungen vorkommen.

Obwohl anzunehmen ist, dass einigen Zeugen und einem vermutlich noch größeren Anteil der Angeklagten nicht geglaubt wird, kommt es außerordentlich selten vor, dass sie vom Richter bereits während der Beweiserhebung explizit beschuldigt werden die Unwahrheit zu sagen. Selbst nach - von außen betrachtet - „eindeutigen" Nachfragen und entsprechend „windigen" Ausflüchten werden Aussagen nur in ganz seltenen Fällen mit Attributen wie unwahr, unglaubwürdig, gelogen und Ähnlichem belegt. ${ }^{16}$ Die beteiligten Juristen, vor allem aber die Richter vermeiden es offensichtlich, eindeutige Einschätzungen zur Beweislage noch während der Beweiserhebung bekannt zu geben - und eben dies scheint einen wichtigen Aspekt ihrer professionellen Kompetenz auszumachen! Die Kompetenzunterstellung liegt deshalb nahe, weil - wie Interviews erweisen - hinsichtlich der Würdigung von Zeugen und ihren Aussagen in den allermeisten Fällen zwischen den juris-

16 Kritische Juristen und Psychologen irritiert dies; siehe etwa ROLF BENDER/ARMIN NACK, Tatsachenfeststellung vor Gericht. 2 Bde., München 1995 oder GERHARD REINICKE, Die Krise der Beweiswürdigung im Strafprozeß oder Über die Schwierigkeit, einem Zeugen nicht zu glauben, in: Monatsschrift fiur Deutsches Recht 8 (1986), 630-637. Sie nehmen die Sache ernst und führen die vermeintliche Leichtgläubigkeit gerade der Richter auf fehlerhafte Alltagstheorien, mangelndes Wissen oder sogar Feigheit zurück. Abhilfe versprechen sie sich durch Forcierung des aussagepsychologischen Problembewusstseins und durch eine größere Entschiedenheit, um nicht $z u$ sagen durch mehr Ehrlichkeit des Gerichts. Wenn man bedenkt, wie lange schon derartige Klagen vorgebracht werden, dann erweisen sich die Richter als eigenartig dickfällige Zeitgenossen. Statt sie nun aber zu "Entscheidungsdeppen" zu degradieren, was einem Sozialwissenschaftler schon überhaupt nicht ansteht, ziehe ich es, dahinter Methode zu vermuten und zunächst einmal nach interaktionsstrukturellen Gründen für die inkriminierte Gutgläubigkeit zu suchen. 
tischen Verfahrensbeteiligten weitgehende Übereinstimmung besteht auch und gerade, wenn es diesbezüglich zu keinen ausdrücklichen Feststellungen und Diskussionen gekommen war. Offensichtlich gelingt es den Akteuren des Gerichts allein schon durch die Art und Weise ihres Fragens in der Situation für alle praktischen Zwecke Glaubwürdigkeit (bzw. Unglaubwürdigkeit) als Tatbestand zu etablieren. ${ }^{17}$

Bezweiflungsprozesse gliedern sich in zwei Phasen: die Phase der allgemeinen Glaubwürdigkeitsprüfung und die Phase der Konstruktion von Unglaubwürdigkeit. Jede Phase ist durch die Verwendung bestimmter Bezweiflungsformate gekennzeichnet. Beide Phasen bauen aufeinander auf. Es muss immer erst die allgemeine Glaubwürdigkeitsprüfung abgearbeitet sein, bevor man zur Konstruktion von Unglaubwürdigkeit übergehen kann. Die Bezweiflungsaktivitäten steigern sich schrittweise im Hinblick auf die Massivität der verwendeten Formate. Unvorbereitete Bezweiflungen von Aussagen führen demgegenüber fast unweigerlich zu Interaktionskrisen und Einsprüchen. Die, soziale Objektivität' einer Befragung beruht anscheinend in der vermeintlichen Zwangsläufigkeit, mit der sich angesichts unbefriedigender Antworten immer massivere Frageformate aufdrängen..$^{18} \mathrm{Zu}$ den Formaten der allgemeinen Glaubwürdigkeitsprüfung zählen in der Reihenfolge ihrer Intensität:

- Fragen unter Verwendung von bestimmten, die Skepsis signalisierenden Partikeln („denn", ,ja“, ,eigentlich“),

- die Kontrastierung mit Normalitätsmodellen,

- der Vorhalt.

Im Laufe der sukzessiven Verwendung solcher Formate verändert sich die pragmatische Wirkung von Fragen. Diese können dann nicht mehr durch Zusatzinformationen, sondern müssen mit Erklärungen, Begründungen oder Rechtfertigung beantwortet werden. Die Frageintention verschiebt sich von der Ermittlung von Information hin zur Prüfung von Kompetenz und Integrität. Dieser Aspekt steht in der nächsten Phase der Bezweiflung, nämlich jener der interaktiven Konstruktion von Unglaubwürdigkeit, dann eindeutig im Vordergrund. Zu den Formaten dieser Phase gehören:

\footnotetext{
17 Wir sprechen im Hinblick auf die hier zum Ausdruck kommende interaktive Leistung der Beteiligten von "kompetenter Skepsis", vgl. WOLFF/MÚLLER, Kompetente Skepsis (Fn. 5).

${ }^{18}$ Insoweit besteht eine Analogie zur Folterpraxis der Inquisition, deren Objektivität ebenfalls in der schrittweisen und sich fein dosiert steigernden Applikation des institutionell vorgesehenen Instrumentariums am „verstockten" peinlich Befragten gründete.
} 
- die Wiederholung bereits einmal gestellter und beantworteter Fragen,

- die Wiederholung der Zeugenbelehrung,

- die Markierung von und die Konfrontation mit ,Freud'schen Fehlleistungen',

- die Frage nach der Gegenversion,

- bestimmte Versionen von Vorhalten (,mundane Rätsel'),

- die Ironisierung.

Eine umfangreiche und sich auf verschiedene Themen und Sachverhalte beziehende Vernehmung kann mehrere solcher Bezweiflungszyklen aufweisen. Auf Phasen, in denen massive Zweifel deutlich gemacht wurden, können ,normale' Befragungsphasen folgen, an die sich wieder Bezweiflungsphasen anschließen und so weiter. Man beginnt bei jedem Durchlauf gleichsam von Neuen. ${ }^{19}$ Allerdings scheint es eine leichte Tendenz zur Beschleunigung und Intensitätssteigerung der Bezweiflung zu geben, obwohl selbst nach sehr intensiven Bezweiflungen eine Befragung mit ,harmlosen' Informationsfragen abgeschlossen werden kann.

Bezweiflungsaktivitäten müssen in der aktuellen Prozesssituation inszeniert werden. Selbst wenn die Glaubwürdigkeit der betreffenden Personen oder die Glaubhaftigkeit ihrer Aussagen in den einschlägigen Akten, in der Anklageschrift oder in den Unterlagen aus einem vorherigen Verfahren eindeutig in Frage gestellt wurden und entsprechende Schlussfolgerungen für alle Beteiligten auf der Hand liegen, kann man nicht gleich mit massiven Bezweiflungsformaten beginnen oder sich gar zu eindeutigen Feststellungen versteigen. ${ }^{20}$ Bei einem solchen Vorgehen wäre insbesondere der Richter immer in Gefahr Verfahrenskrisen, zum Beispiel einen Befangenheitsantrag, zu provozieren. Daher übernimmt der Staatsanwalt, für den diese Gefahr nicht gilt, oft seinen Part, oder doch die massiveren Bezweiflungen. Richter und Staatsanwalt bilden bei der sukzessiven Verschärfung der Bezweiflung nicht selten ein regelrechtes ,Team' mit eingespielter Arbeitsteilung.

Zurückhaltung ist angesichts ihrer Platzierung am oberen Ende der Bezweiflungsskala gerade für ironische Äußerungen geboten. Wir haben eine Verhandlung beobachtet, in der der Richter die Angeklagte schon ganz zu Beginn der Vernehmung ironisiert hatte. Dies führte

${ }^{19}$ Diese Form der Konsistentunterbrechung erlaubt es zudem die Frage der Glaubhaftigkeit von Aussagen von der Problematisierung der Glaubwürdigkeit der Person zu trennen.

${ }^{20}$ Diese ist gleichsam die interaktionistische Reformulierung und Präzisierung des Mündlichkeitsprinzips der Strafprozessordnung ( $\$ 261$ StPO). 
prompt dazu, dass der Verteidiger drohte, einen Befangenheitsantrag einzubringen. Der Befangenheitsantrag wurde zwar nicht mit dem Verweis auf diese Episode begründet, wohl aber, wie sich aus einem späteren Gespräch mit dem Verteidiger ergab, durch diese Szene motiviert. ${ }^{21}$

Die endgültige Feststellung von Unglaubwürdigkeit geschieht in der Regel stillschweigend. Sie erfolgt entweder durch Verfahrensentscheidungen, die implizit die Unbrauchbarkeit der betreffenden Aussage für den gerichtlichen Entscheidungsprozess ausdrücken (z. B. durch Verfahrenseinstellung mit oder ohne Auflagen), durch einen Freispruch, der mit "letzten Zweifeln" begründet wird und einfach durch bloße Nichtbeachtung. Diese Zurückhaltung gegenüber expliziten und transparenten Einschätzungen der Glaubwürdigkeit ist verständlich, wenn man bedenkt, dass ausdrückliche Beschuldigungen von Zeugen die Unwahrheit gesagt zu haben, zwangläufig ein juristisches Nachspiel in Form eines Verfahrens wegen uneidlicher Falschaussagen oder gar wegen Meineids haben. Aber selbst gegenüber den Angeklagten, die keiner Wahrheitspflicht unterliegen, halten sich zumindest die Richter mit entsprechenden Qualifizierungen auffällig zurück. Ein gewisses Maß an Intransparenz über das, was gerade im Verfahren der Fall ist, scheint somit zumindest von den Richtern nicht nur in Kauf genommen, sondern sogar angestrebt zu werden. ${ }^{22}$

\subsection{Sequentielle Aspekte der Ironie}

Die Ironie kommt zwar wie der Blitz, aber nie aus dem heiteren Himmel. Ironisierungen scheinen nur in bestimmten kleinräumigeren interaktiven Konstellationen realisiert zu werden. Sie benötigt immer einen ,Aufhänger', wobei die Aufhänger vom Objekt der Ironie geliefert werden müssen. ${ }^{23}$ Folgende Grundstruktur ist für ironische Episoden in unserem Material charakteristisch: Ironisierende Äußerungen sind immer zweite Äußerungen. Der Ironisierende reagiert auf eine andere

\footnotetext{
${ }^{21}$ Um dieses Risiko wusste schon Quintilian, der im Gebrauch der Ironie einen schwerwiegenden und hinreichenden Beleidigungsgrund erkannte und deshalb Vorsicht empfahl.

22 Diese Beobachtung korrespondiert mit der These Luhmanns, wonach die Gewissheit, „dass eine Entscheidung getroffen wird und die Ungewissheit, welche Entscheidung es sein wird", neben dem eigenen Interesse am Thema die Beteiligten zu sachgerechter Mitwirkung am Verfahren motiviert. Insbesondere die Ungewissheit des Ausgangs sei verfahrenswesentlich; vgl. LUHMANN, Legitimation durch Verfahren (Fn. 1), 50.

${ }^{23}$ Dies ist ein erster Hinweis darauf, dass und warum eine sprechakttheoretisch argumentierende Konzeption von Ironie problematisch ist. Es ist nämlich nicht die Äußerung als solche, sondern die sequentielle Einbettung, die eine Äußerung (ob sie nun ironisch gemeint war oder nicht), zu einer wirklichen, d. h. interaktiv geglückten Ironie werden lässt.
} 
Aussage, nämlich auf eine des späteren Ironisierungsobjektes. Dabei handelt es sich in praktisch allen Fällen um den unmittelbar vorhergehenden Redezug. Typischerweise ist dies eine Äußerung, die als Einwand, Gegenargument, Behauptung, Klarstellung, Unterbrechung oder Ähnliches formuliert ist und/oder vom Ironisierenden retrospektiv als solche behandelt wird. Ironische Bemerkungen setzen bevorzugt an vermeintlichen, Vergehen' gegen das Verfahrenssystem und gegen die darin herrschenden Verhaltenserwartungen an. Erst wenn eine derartige Differenz zwischen den Erwartungen an den Zeugen und seinem aktuellen Verhalten kommunikativ etabliert ist, lässt sich eine Ironie erfolgreich platzieren.

Zielscheibe der Ironie ist die Person, die diese letzte Äußerung getan hat und nicht jemand, der oder die in dieser Äußerung angesprochen wurde. Die ironische Bemerkung bezieht sich dann zwar oft direkt auf inhaltliche Aspekte der vorherigen Einlassung; dies muss aber nicht so sein. Auch zurückliegende Äußerungen und Verhaltensweisen des Ironieobjekts können unter bestimmten Umständen ironisch aufgespießt werden. So kann etwa ein Staatsanwalt einen früheren Gesprächsausschnitt (z. B. einen aus der richterlichen Vernehmung) noch einmal rekapitulieren und daran dann die eigene(n) ironische(n) Bemerkung(en) anschließen.

Diese allgemeinen Beobachtungen lassen sich anhand unseres empirischen Materials noch präzisieren. Unser erstes Beispiel stammt aus einem Verfahren wegen Meineids und versuchter Strafvereitelung.

((Dem Angeklagten Bracke wird vorgeworfen, zugunsten seines Freundes Tupalla in einem anderen Prozess wegen Trunkenheit im Straßenverkehr eine falsche Aussage unter Eid und eine uneidliche Falschaussage gemacht $\mathrm{zu}$ haben. Im jetzigen Verfahren hatte Tupalla bereits als Zeuge ausgesagt, Bracke habe am Steuer gesessen. Er selbst habe auf dem Beifahrersitz mit zurückgedrehter Lehne vor sich hingedöst, während sie von einem Polizeiwagen verfolgt wurden. Im Transkriptionsausschnitt geht es um die Frage, ob die Polizeibeamten zweifelsfrei erkennen konnten, dass nicht Bracke, sondern Tupalla am Steuer saß.))

$S \quad$ ja also (0.2) da hab ich an die beidn noch ma eine Frage.

$R$ ja:

$\mathrm{S} \quad=$ dieselbe praktisch.

$$
\text { (2.6) }
$$

S un zwar bezieht sich das nämlich darauf, (0.8) wie die beiden Herrn, (0.4) sich: (1.0) im Profil ähnlich sehn, und:dh (0.4) wie sie von vorne, $(0.4)$ oder auch so wenn se sich bewegn,

(.) welchn Eindruck sie machn da $\sin (d)$ se sich gar nich mehr so ähnlich, (1.0) 
R ne:. also jetz wenn ich (se) vor mir sehe, (0.6) aber da sieht ma ma wieder was:: e::

$\mathrm{S}$ ma muss die Leute sich::

$\mathrm{R} \quad \mathrm{ja}{ }^{\circ}$
(0.4)

$\mathrm{S}$ bewwegn sehn. ma muss sie

$(0.2)$

$\mathrm{R} \quad(\mathrm{n}) \mathrm{ja}$
(0.4)

$S$ ein

$Z \rightarrow>$ (er) im Auto ham wir doch gesessn un ham (e) umgel(a)

$S$-> grade das wollt ich jetzt die beidn Herrn noch ma fragen das könn wer alles klärn Herr ((langsam, gewichtig)) Tup:alla hh

R ((abgehackt))

so:. dann e:

$(0.2)$

S (( schneller, schärfer werdend))

$\rightarrow \quad$ sie kön das ja im übrign gar nich wissen. sie war $n$ ja abgetaucht; sie hattn ja ihrn Rücksitz zurückgestellt un ham die Polizeibeamtn gar nich wahrgenomn; nech, nur noch ma um Ihnn Sie daran zu erinnern, welche Geschichte Sie hier vertretn müssn.

(1.0)

(( Geräusch))

(0.4)

$\mathrm{R} \quad$ den Herrn Grell bitte erst noch mal: hh

Verfahren Bracke, Zeugenaussage Tupalla, 20-brac5.txt, 40/41

In diesem Ausschnitt finden wir die typische kommunikative Grundstruktur von Ironisierungen wieder: Der Zeuge Tupalla ist in einem vorherigen Verfahren wegen Trunkenheit im Straßenverkehr verurteilt worden. Seine und die Aussagen des jetzigen Angeklagten Bracke wurden damals ausdrücklich als unglaubwürdig eingeschätzt. In einem Berufungsverfahren vor dem Landgericht wurde dieses Urteil bestätigt. Dies lässt seine Glaubwürdigkeit auch in diesem Verfahren als problematisch erscheinen, wie eine Reihe entsprechender Bezweiflungsaktivitäten während der bisherigen Vernehmung schon gezeigt hatte. Von daher ist er von seiner sozialen Kategorisierbarkeit als ,unglaubwürdig' her bereits ein mögliches Bezugsobjekt ironischer Bemerkungen. 
In dieser Szene nun setzt der Staatsanwalt (S) von gelegentlichen $\mathrm{Zu}$ stimmungsbekundungen und Ergänzungen der Richterin (R) begleitet zu einer Frage an, in die sich später der Zeuge mit dem Versuch einer Richtigstellung einschaltet (,... im Auto ham wir doch gesessen ..."). Diese Äußerung wird vom Staatsanwalt als Unterbrechung seiner eigenen Äußerung und implizit als eine Verletzung der Zeugenrolle markiert (,Gerade das wollt ich jetzt die beiden Herren noch mal fragen ..."). Nachdem der Einmischungscharakter und die Voreiligkeit der Äußerung des Zeugen noch ein weiteres Mal betont wurde, folgt die ironische Bemerkung: „Nur noch mal, um Sie daran zu erinnern, welche Geschichte Sie hier vertreten müssen."

Ironische Äußerungen können nur dann glücken, wenn sie ganz spontan aus einer bestimmten Situation heraus erfolgen. Es darf nicht der Eindruck des Gewollten und Gemachten aufkommen. Dies ist aber nur zu erreichen, wenn sie punktgenau in den sequentiellen Ablauf der Interaktion eingepasst wurden. Der Staatsanwalt ,musste' so gesehen die ironische Äußerung unmittelbar nach dem Einwurf des Zeugen an diesem Ort anbringen. Hätte er erst einem oder mehreren Redezüge verstreichen lassen, wäre die pragmatische Wirkung seiner Äußerung eine ganz andere gewesen. In einer Reihe von Beispielen wird dieser unmittelbare Situationsbezug durch Ausdrücke wie "gerade das", "genau" oder ,jetzt" noch einmal ausdrücklich markiert.

Angeklagte wie Zeugen sind gesprächsstrukturell gesehen besonders verletzlich für ironische Attacken. Dies nicht nur, weil eine etwaige Gegenironie oder gar eine meta-sprachliche Beschwerde dagegen große Schlagfertigkeit erfordern würden. Wichtiger erscheint, dass derartige Äußerungen nicht zu den kategoriegebundenen Aktivitäten von Zeugen passen. Typisch für die institutionelle Kommunikation vor Gericht ist es ja, dass alle ihre Abschnitte so strukturiert sind, dass sich die Zeugen und in abgeschwächter Weise auch die Angeklagten in der Situation der Reagierenden befinden. Alles das, was sie zur gerichtlichen Kommunikation beitragen, können sie darüber hinaus legitimer Weise nur im Rahmen bestimmter Äußerungsformen und zu bestimmten Gelegenheiten tun: nämlich nach einer Initiative des Richters (oder einer von ihm bevollmächtigten Person) und im Rahmen von Antworten und Sachverhaltsschilderungen. Der Versuch einer Reaktion, Kommentierung bzw. Zurückweisung der Ironie ist besonders begründungsbedürftig und damit interaktiv aufwendig. Die Zurückweisung der Ironie würde ja nicht nur den Inhalt und die Form der Frage, sondern darüber hinaus die Intentionen und den Status des Fragestellers betreffen. Der Zeuge würde damit das Verhörverhalten des Richters, Staatsanwalt oder Verteidigers bzw. die Prozessführung als solche 
beanstanden und so unter Umständen eine gravierende interaktive Krise mit schwer absehbaren Konsequenzen riskieren.

Angesichts dieser interaktionsstrukturellen Lage ist es vermutlich kein Zufall, dass wir unmittelbar nach der Äußerung des Ironiesubjektes oft eine Schweigephase beobachten, die sich in den uns vorliegenden Verfahren bis zu 25 Sekunden Länge hinziehen kann. Wird auf ein Weiterreden trotz ausdrücklicher Anrede zunächst verzichtet, spricht man in konversationsanalytischer Terminologie von einer Redezugvakanz ${ }^{24}$. Es bestünde für den Zeugen oder Angeklagten an dieser Stelle nicht nur die Möglichkeit einer Reaktion, sondern sogar eine entsprechende konditionelle Relevanz dahingehend. Thr Schweigen erhält den Charakter einer aktiven Nicht-Handlung und bekommt eben dadurch Bedeutung. Eine Redezugvakanz kann man zwar nicht mit einem Schuldeingeständnis oder dem Einräumen einer Falschaussage gleichsetzen. Dazu ist ein Schweigen zu viel sagend. Diese Vagheit scheint das Gericht indes nicht zu stören, im Gegenteil. Das Schweigen lässt für die Beteiligten Interpretationsspielräume und Handlungsmöglichkeiten offen, die sich im weiteren Verlauf der Verhandlung nutzen lassen. Dafür spricht, dass derartige Schweigephasen vom Richter als den zuständigen Verfahrenswalter mitunter sehr lange offen gehalten werden, bevor er seinerseits einen Abschluss bzw. eine Fortsetzung der Befragung einleitet. Er enthält sich üblicherweise auch jeglicher Kommentierung. Ironische Episoden gewinnen durch diese - scheinbare Folgenlosigkeit einen eigenartig insulären und herausgehobenen Charakter im Situationsablauf.

Ein zweiter Blick auf das angeführte Beispiel demonstriert, wie kunstvoll und orchestriert die Beteiligten dabei vorgehen. Genau besehen haben wir es hier nämlich mit einer stillschweigenden Kooperation zwischen Staatsanwalt und Richterin zu tun

Z (er) im Auto ham wir doch gesessn un ham (e) umgel(a)

$\mathrm{S}$ grade das wollt ich jetzt die beidn Herrn noch ma fragen

das könn wer alles klärn Herr ((langsam, gewichtig)) Tup:alla hh

R ((abgehackt))

$\rightarrow$ so: dann e:

$(0.2)$

S (( schneller, schärfer werdend))

$\rightarrow$ sie kön das ja im übrign gar nich wissen. sie war $n$ ja abgetaucht; sie hattn ja ihrn Rücksitz zurückgestellt un ham die

24 JORG R. BERGMANN, Schweigephasen im Gespräch, in: HANS-GEORG SOEFFNER (Hrsg.), Beiträge zu einer empirischen Sprachsoziologie. Tübingen 1982, 143-184. 
Polizeibeamtn gar nich wahrgenomn; nech, nur noch ma um Ihnn Sie daran zu erinnern, welche Geschichte Sie hier vertretn müssn.

(1.0)

(( Geräusch))

R-> den Herrn Grell bitte erst noch mal: hh

Verfahren Bracke, Zeugenaussage Tupalla, 20-brac5.txt, 40/41

Die ironische Äußerung erfolgt, nachdem die Richterin bereits ihrerseits mit ihrem Beitrag begonnen und durch seine Gestaltung einen Abschluss eines Verfahrensabschnittes sowie einen Themenwechsel hin zur Befragung der Polizeizeugen mit "so:. dann eh" eingeleitet hatte. 25 Auffällig ist, dass der Staatsanwalt der Richterin mit seiner ironischen Äußerung ins Wort fällt, was diese dadurch, dass sie nach deren Beendigung und einer Pause von einer Sekunde ihre angefangene Äußerung weiterführt, nachträglich akzeptiert. Diese Intervention wird durch die Richterin demnach nicht als Unterbrechung ihrer Äußerung behandelt. Sie tut zumindest nichts, um die kommunikative Wirkung der ironischen Bemerkung zu schmälern. Indem sie damit eine ihr durchaus mögliche, ja sogar von der Beteiligtenstruktur des Gerichtsverfahrens her erwartbare Handlungsoption (nämlich: Monieren einer Unterbrechung ihrer Verhandlungsführung) nicht wahrnimmt, unterstützt und verstärkt sie in gewisser Weise sogar die Wirkung dieser Attacke.

Dieser Abschnitt ist ein Beleg für eine weitere Regelmäßigkeit in unserem Material: dass nämlich das Publikum (also alle jene Verfahrensbeteiligten außer dem Ironiesubjekt und dem Ironieobjekt) keinen aktiven Part in der Ironiesequenz spielt. Weder reagiert die Richterin auf den ausbleibenden Redezug des Zeugen, indem sie ihn etwa zu einer Antwort auffordert, noch moniert sie die offensichtliche Unterbrechung durch den Staatsanwalt. Stattdessen geht sie einfach zu einem neuen Befragungsthema weiter. Das Beispiel zeigt somit, dass die Nicht-Reaktion der Richterin keine bloße Passivität darstellt, sondern dass wir es hier mit einer aktiven Zurückhaltung, d. h. der erkennbaren Nichtwahrnehmung von offensichtlichen Optionen zu tun haben.

\footnotetext{
25 Äußerungen wie „so“ und „dann” dienen in Mehrpersonengesprächen gewöhnlich als Markierer zur Gesprächsstrukturierung. In dem Maße, in dem immer nur Vertreter einer bestimmten Teilnehmerkategorie sich ihrer bedienen, lassen sie sich als Ausdruck situationsinterner Dominanzverhältnisse verwenden, vgl. CHRISTOPH MEIER, Arbeitsbesprechungen. Interaktionsstruktur, Interaktionsdynamik und Konsequenzen einer sozialen Form. Opladen 1997.
} 


\section{Merkmale ironischer Äußerungen}

Schauen wir uns nun die ironische Äußerung selbst an. Was macht sie als ironisch gemeint oder zumindest als ,ironiefähig“ erkennbar? Einige analytische Beobachtungen lassen sich am folgenden Ausschnitt der uns nun schon bekannten Szene machen.

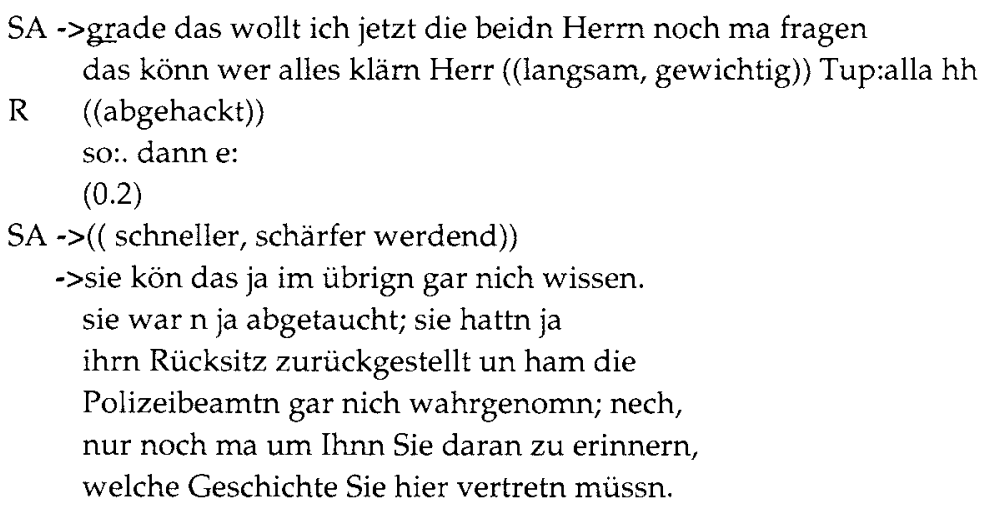

Verfahren Bracke, Zeugenaussage Tupalla, 20-brac5.txt, 40/41

Der Staatsanwalt unterstellt offenbar, dass der Zeuge gerade etwas behauptet hat, was er nach seiner früheren Darstellung, wonach er vor sich hingedöst und die Beamten nicht wahrgenommen habe, gar nicht wissen kann. Auf diesen Widerspruch bezieht sich seine Bemerkung. Dabei fällt die betonte Fürsorglichkeit des Staatsanwalts in Bezug auf den Zeugen auf. Nimmt man seine Äußerung wörtlich, dann "hilft" er dem Zeugen dabei, eine bestimmte Geschichte, nämlich seine bislang vorgebrachte, weiterhin vertreten zu können. Er tut dies, indem er ihn daran erinnert, dass er etwas nicht wissen könne, es folglich auch hier nicht darstellen dürfe, wenn seine Einlassung konsistent bleiben soll.

Eine solche wörtliche Interpretation des Sinns der Äußerung des Staatsanwalts kann aber nicht richtig sein, da - wie jeder weiß - die Pflicht des Zeugen im Gegenteil darin besteht, die Wahrheit mitzuteilen, d. h. eben keine "Geschichte" zu erzählen. Hinzukommt, dass der Staatsanwalt gleichsam zum gegnerischen Team gehört, aber an dieser Stelle so tut, als ob dies gar nicht der Fall sei und er sich unvoreingenommen, ja sogar helfend an den Zeugen wenden würde. Die ironische Pointe, also die Auflösung dieses Widerspruchs, läuft darauf hinaus, dass der Zeuge eben nicht die Wahrheit sagt, sondern versucht eine (erfundene) Geschichte zu vertreten. 
Ganz ähnlich ist der folgende Fall gebaut:

$S$ (z) muss ich doch noch ma genau fragn. wie war s denn nun wirklich.

$\mathrm{Z}$ jas

S oder;kann's gibt's vielleicht noch ne dritte Möglichkeit die in Betracht kommt; die Ihnn möglicherweise noch gar nicht einge= falln is.

?? ((Räuspern))

Z nja s wird wohl schon so gewesn sein dass ( ) (s) Auto aus der Einfahrt rausfahrn wollte damith halt

Verfahren Bracke, Zeugenaussage Tupalla, 20-brac5.txt, 25/26

Der Staatsanwalt stellt zunächst eine Nachfrage (,wie wars denn nun wirklich?"). Diese Frage setzt voraus, dass es nur eine wirkliche Version der Geschichte gibt und dass der Angeklagte diese kennt, aber bislang nicht vorgebracht hat. Dies wiederum impliziert, dass der Staatsanwalt an die (von diesem behauptete) Erinnerungsschwäche des Zeugen nicht glaubt. Solche Fragen nach der Wahrheit oder danach, wie es "wirklich war", beinhalten immer die Unterstellung von Unglaubhaftigkeit der vorherigen Einlassungen. In einem eigenartigen Kontrast zu dieser Frage nach der Wahrheit steht die darauf folgende Äußerung des Staatsanwalts, in der dieser fragt, ob es noch eine dritte Möglichkeit gäbe, die dem Zeugen noch nicht eingefallen sei. Wiederum ist der Zeuge mit einer ,unmöglichen Frage' konfrontiert. Ein glaubwürdiger Zeuge stellt ein Ereignis wahrheitsgemäß dar, und zwar in jeder seiner Einlassungen. Es kann somit nicht seine Aufgabe sein, sich eine ihm genehme Version einfallen zu lassen und dann, wenn Akzeptanzschwierigkeiten auftreten, weitere Alternativversionen nachzureichen. Dies würde nur bei unglaubwürdigen Zeugen zutreffen.

Angriffspunkte ironischer Bemerkungen sind neben der Konstatierung von Aussagewidersprüchen die Feststellung der Unwahrscheinlichkeit einer Darstellung. Letzteres betrifft Darstellungen, die nach Ansicht eines professionellen Prozessbeteiligten im Gegensatz stehen zu allgemein geteilten Erwartungen von Folgerichtigkeit, Normalität und Vernunft. Das Vorliegen einer statistischen Unwahrscheinlichkeit reicht hingegen für eine grundlegende Bezweiflung der Glaubhaftigkeit der Aussage bzw. der Glaubwürdigkeit der jeweiligen Person nicht aus. 
Vielmehr muss schon eine sozial relevante Unwahrscheinlichkeit in der Situation etabliert werden. Der übliche Weg besteht darin, die betreffende Aussage mit bestimmten Normalformerwartungen (wie bestimmte Situation prototypisch ablaufen), kategorialen Unterstellungen (was man von bestimmten Kategorien von Personen erwarten kann) oder Rationalitätsunterstellungen (an was man sich üblicherweise erinnert) zu kontrastieren.

Solche sozial relevanten Unwahrscheinlichkeiten bloß zu konstatieren scheint für eine massive Bezweiflung aber noch nicht zu genügen. Die Ironisierung erweist sich hier als ein kommunikatives Hilfsmittel, um aus einer bloß unwahrscheinlichen eine zweifelhafte, $d$. h. eine die mangelnde Glaubwürdigkeit der betreffenden Person implizierende und demonstrierende Aussage werden zu lassen. ${ }^{26}$ Ein Aspekt ironischer Äußerungen besteht entsprechend darin, vorherige Darstellungen der Ironieobjekte $\mathrm{zu}$ reformulieren und sie dabei erkennbar zu überziehen..$^{27}$

((Der Zeuge, ein Handelsvertreter, hatte unmittelbar vorher behauptet, er habe dem Angeklagten, einem Drogenabhängigen, 5000 Mark für ein geschäftliches Unternehmen geliehen, ohne genau zu wissen, wofür das Geld bestimmt war. Einen Teil dieses Geldes habe er sich von seiner heutigen Frau geliehen. Hierauf bezieht sich die erste hier zitierte Äußerung des Richters. Der Richter folgert, dass sich der Zeuge auch noch gegenüber einer anderen Person verpflichtete. Nachdem der Zeuge dies bestätigte, folgt die ironische Bemerkung des Richters))

$R \quad$ und sie haben sich quasi anderen gegenüber auch

noch mal verpflichtet um ihm das Geld zu ihm das Geld zu besorgen.

$\mathrm{Z}$ richtig.

$\mathrm{R}$-> dafür gibt s keine andere Erklärung als dass sie ihm das:

auf $\operatorname{sein}(\mathrm{e})$

(1.0)

seine nette Art hin

(0.4)

zur Verfügung stellen.

(3.0)

Z ${ }^{\circ}(\text {.hh })^{\circ}$ e:hff:: ja. w wo hätt er s Geld sonst herkriegen;

Verfahren Krapp, Zeugenaussage Werner, 06krap1a, 25/26

${ }^{26}$ Wir sprechen von Glaubwürdigkeit, weil sich Ironisierungen nie nur auf die Aussage, sondern immer auch und vor allem auf deren Urheber beziehen.

27 Analog der Übertriebenheit der angetragenen ,Fürsorglichkeit' im vorletzten Ausschnitt. 
Der Richter spitzt die Darstellung des Zeugen dahingehend $z u$, dieser habe dem Angeklagten wohl das Geld "auf seine nette Art hin" zur Verfügung gestellt. Wenn jemand, noch dazu, wenn er kaufmännisch tätig ist, einer anderen Person lediglich auf seine nette Art hin 5.000 Mark leiht, wäre dies außergewöhnlich naiv. Der ironische Effekt wird dadurch zu erzielen versucht, dass der Zeuge implizit dazu eingeladen wird, sich selbst als naiv zu bezeichnen, um der anderen Interpretationsmöglichkeit („unglaubwürdig“) zu entgehen. Ironische Äußerungen enthalten also widersprüchliche Implikationen. Man kann sie daher nicht wörtlich nehmen ohne mit bestimmten Normalitätsannahmen zu kollidieren.

Dieser Umstand erschwert es zusätzlich auf ironische Fragen oder Bemerkungen direkt zu reagieren. Geschickt gewählte Kontraste bringen den Ironisierten in eine Art Doppelbindung: egal, ob und wie er reagiert - seine Reaktion ist problematisch. Reagiert er so, als ob nichts gewesen sei, demonstriert er seine Naivität. Reagiert er nicht, so ist ihm diese Nicht-Reaktion als, offizielles Fehlen' zurechenbar. Wenn er andererseits die Zumutung anspricht, die die ironische Äußerung impliziert, überschreitet er das für ihn im Aktivitätstyp der Zeugenbefragung strukturell vorgesehene Handlungsrepertoire. Typisch für die kommunikative Wirkung aller dieser Formate ist es somit, dass sie eben nicht auf Antworten aus sind, sondern den Zeugen, sprachlos' und eben diese Sprachlosigkeit für alle Beteiligten sichtbar machen. Ironisierungen gestatten es also, Aufmerksamkeit zu fokussieren und Hinweise zu geben, ohne zu endgültigen Festlegungen greifen zu müssen.

Kontraste und Übertreibungen sind für sich genommen noch keine eindeutigen Indikatoren für Ironie; vor allem keine Indikatoren, die allein für Ironie spezifisch wären. Daher ist semantisch letztendlich nicht $\mathrm{zu}$ erfassen, ob eine Äußerung ironisch oder ernsthaft gemeint war. Im Grunde stehen wir Konversationsanalytiker damit vor einem ähnlichen Problem wie die Hörer einer ironischen Äußerung in der Situation. Weil die entsprechenden Signale vage und uneindeutig sind (und um der Wirkung der Ironie Willen sein müssen!), reichen sie nicht aus, um Ironie als soziale Tatsache eindeutig festzustellen. Es stellt sich nun die Frage, ob die Beteiligten bei ihren entsprechenden Interpretationsprozessen zumindest auf gewisse formale Merkmale ironischer Äußerungen zurückgreifen können.

In der Literatur gibt es vielfältige Vorschläge zur Feststellung und Systematisierung derartiger Ironiesignale. Mimische, gestische und intonatorische Modulationen wie Augenzwinkern, Räuspern, eine emphatische Stimme oder die Häufung bombastischer Ausdrücke, gewag- 
te Metaphern, überlange Sätze, Wortwiederholungen und ,hörbare' Anführungszeichen werden als Kandidaten genannt. Alles dies kommt in unserem Material vor, ohne dass dabei freilich eine durchgängige Systematik erkennbar wäre.

Der schon mehrfach zitierte Gesprächsausschnitt zeigt uns einige der auf der averbalen Ebene besonders häufig zu beobachtenden Merkmale ironischer Bemerkungen. An diesen Äußerungen fällt eine in aller Regel gegenüber vorhergehenden Abschnitten veränderte Tonlage (in unseren Beispielen meist lauter) und Sprechgeschwindigkeit auf. Hinsichtlich der Sprechgeschwindigkeit konnten wir allerdings keine eindeutige Richtung beobachten. Zwar steigert sich diese in der Mehrzahl der Fälle; es kommen aber durchaus auch auffällige Verzögerungen und Rhythmisierungen (,Wort-für-Wort-Sprechen“) vor. In jedem Falle wird so die betreffende Äußerung aus dem Verlauf der übrigen Interaktion wahrnehmbar herausgehoben. In dieselbe Richtung weist der phasenweise Gebrauch von Dialekt- und Slang-Varianten ( "könn' wer").

Eine Form der Markierung findet sich allerdings fast durchgängig: Dies betrifft das Vorkommen von Ausdrücken wie "nur noch mal”, „im Übrigen", „nur um", "möglicherweise", "streng genommen", „im GroBen und Ganzen", „eigentlich", „ja" u. Ä. Sie alle gehören zu einer Klasse von sprachlichen Phänomenen, die in der linguistischen Pragmatik als ,hedges' bezeichnet wird. Darunter versteht man ein Partikel, ein Wort oder eine Floskel,

"that modifies the degree of membership of a predicate or noun phrase in a set; it says of that membership that it is partial, or true only in certain respects, or that it is more true and complete than perhaps might be expected." 28

Solche "Schutzhecken" haben die kommunikative Funktion, potentiell unhöfliche und unkooperative Implikationen von Äußerungen abzuschirmen. Durch die Verwendung derartiger Ausdrücke in ironischen Äußerungen wird also einerseits das Bedrohungspotential der Ironie markiert, zugleich aber einer feindseligen oder entrüsteten Reaktion darauf vorgebaut.

Insgesamt gesehen wird man der Feststellung von Lapp beipflichten müssen, dass der Versuch, Ironie allein über die semantische Analyse

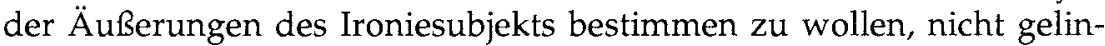
gen kann. ${ }^{29}$ Praktisch alles könnte Ironie signalisieren. Die Rede von "ironischen Sprechakten" ist demnach irreführend. Die einzige Regel, die sich hinsichtlich der Markierung ironischer Äußerungen angeben

\footnotetext{
28 Penelope BROWN/Stephen LeVInSON, Politeness. Some Universals in Language Usage. Cambridge 1987, 145.

${ }^{29}$ Vgl. EDGAR LAPP, Linguistik der Ironie. Tübingen 1992.
} 
lässt ist, dass sie markiert sind, und dass den Ironiesubjekten, um dies zu erreichen, ein breites Repertoire von Möglichkeiten zur Verfügung steht.

Markiert wird freilich weniger der ironische Charakter der Äußerung (dies würde ein eindeutiges Entsprechungsverhältnis von Bedeutung und Äußerung voraussetzen), sondern eher der Umstand ihrer Besonderheit und interaktiven ,Delikatesse'. Somit können zwar sprachliche und außersprachliche Signale dem Hörer ein ironisches Verständnis erleichtern. Das analytisch Entscheidende ist aber, dass solche Signale uneinheitlich und auch nicht in jedem Falle anzutreffen sind. Die linguistische Analyse kann deshalb niemals voraussagen, ob eine Äußerung als ironisch angesehen wird. Letztlich wird immer erst durch die Reaktion der Rezipienten darüber entschieden, ob ein Ironieversuch glückt. ${ }^{30}$ Eine Konversationsanalyse der Ironie wird sich (im Gegensatz zur sprechakttheoretischen Zugangsweise) deshalb nicht auf die Analyse der Äußerungen des Ironiesubjekts beschränken (wobei der Analytiker letztendlich nur auf sein, wie auch immer hermeneutisch geschultes Vorverständnis zurückgreifen könnte), sondern rekonstruieren müssen, wie die Gesprächsteilnehmer selbst diese Äußerung analysieren und ihrem Sinn damit die Eigenschaft einer sozialen Tatsache verleihen.

Sequenzanalytisch gesehen würde der Versuch einer Ironisierung immer dann misslingen, wenn alle Rezipienten durch die Art und Weise ihrer Reaktion zu erkennen geben, dass sie die betreffende Äußerung ernst nehmen ohne die Verspottung zu erkennen bzw. erkennen zu wollen. Hier entsteht allerdings in Bezug auf unseren Gegenstand Gerichtsverfahren eine paradoxe Konstellation: Ganz offensichtlich legen sich hier die in Frage kommenden Rezipienten besondere Beschränkungen hinsichtlich der Deutlichkeit ihrer Reaktionen auf.

Selbst wenn die Ironie und damit einhergehende massive Infragestellung eines Zeugen bzw. Angeklagten und seiner Aussagen für alle Beteiligten subjektiv eindeutig sein mag, so sind doch ihre Reaktionsmöglichkeiten in der gerichtlichen Interaktionssituation grundsätzlich,

\footnotetext{
30 Dies entspricht einer grundlegenden konversationsanalytischen Einsicht: "Conversation is informed by the general assumption - common to both speakers and hearers - that utterances which are placed immediately next to some prior are to be understood as produced in response to, or, more loosely, in relation to that prior. This assumption provides a framework in which speakers can rely on the positioning of what they say to contribute to the sense of what they say as an action. This assumption that adjacent utterances are, whereever possible, to be heard as related was formulated by Sacks ... as a fundamental ordering principle for conversation and he summarized it as the general finding that a turn's talk will be heard as directed to a prior turn's talk, unless special techniques are used to locate some other talk to which it is directed'." JOHN HERITAGE, Garfinkel and Ethnomethodology. Cambridge 1984, 261.
} 
wenn auch in unterschiedlicher Weise, eingeschränkt. Wir haben in keinem Fall beobachtet, dass sich einer der Beteiligten, vor allem nicht die ironisierte Person selbst, ausdrücklich auf den Sachverhalt der Ironisierung bezieht (etwa in Gestalt einer Beschwerde über die situative Unangemessenheit humoriger Aussagen). ${ }^{31}$ In der Konversation zwischen Vertretern des Gerichts und den Ironieobjekten ist, zumindest gilt dies für unser Material, die ironische Komponente der Kommunikation gänzlich ,exkommuniziert'. Fiktiv wird von allen Beteiligten so getan, als ob die Situation ihren ernsthaften Charakter zu keiner Zeit verloren hätte. Das zeigen die Reaktionen der Ironisierten, ebenso wie die Unterdrückung offener Belustigung der anderen Beteiligten während der Befragung. In keinem Fall aus unserem Material kommt es zu lautem Lachen oder ähnlichen Kundgaben des Amüsiertseins. Auch der Fall, dass die ironisierten Personen ihrerseits in eine nicht-ernsthafte Modalität wechseln - etwa indem sie Gegenironien anbringen - findet sich nicht. ${ }^{32}$

Sicherlich beruht die besondere Eleganz ironischer Kommunikation grundsätzlich nicht nur darin, dass sie gleichsam beiläufig daherkommt, sondern auch, dass sie in ähnlich beiläufiger Weise von den Beteiligten goutiert wird. Gerade die ,feine' und treffsichere Ironie verträgt keine allzu deutlichen Reaktionen der anderen Beteiligten. Beide Seiten müssen sich in ihrer Zurückhaltung ergänzen. Das Problem gerade für die ,feine' Ironie liegt nun darin, dass sowohl die ironisierende Äußerung wie die Reaktionen der Rezipienten aufgrund ihres geringen Explizitheitsgrades leicht übersehen oder missverstanden werden können. ${ }^{33} \mathrm{Ab}$ einem gewissen Grad der Sublimität weiß keiner mehr so

31 Diese Zurückhaltung gilt nur für das gerichtliche Interaktionssystem. In Interviews über die entsprechenden Verhandlungen erzählten die Beteiligten sehr wohl über das Vorkommen wie über die Treffsicherheit ironischer Bemerkungen. Auch wurden hier die emotionale Beteiligung (Wut, Entrüstung oder Belustigung), sowie dadurch motivierte prozessstrategische Konsequenzen (Stellen eines Befangenheitsantrags, Verzicht auf eigene Befragung des Zeugen oder Angeklagten, ,Fallenlassen' des Zeugen) deutlich zum Ausdruck gebracht.

32 Wie dies in Alltagsgesprächen gang und gäbe ist (vgl. HELGA KOTTHOFF, Ironie in Privatgesprächen und Fernsehdiskussionen, in: INKEN KEIM/WILFRIED SCHÜTTE (Hrsg.), Soziale Welten und kommunikative Stile. Tübingen 2002, 445-473).

${ }^{33}$ Man könnte diesbezüglich die Interaktionsordnung des Gerichts als einen partiell geschlossenen „Bewusstheitskontext" bezeichnen. Die Betroffenen wissen in vielen Fällen sehr wohl, welches Spiel mit ihnen hier getrieben wird, können dies aber aufgrund der Interaktionsordnung der gerichtlichen Vernehmung von sich aus nicht zum Ausdruck zu bringen. In der Terminologie von Glaser/Strauss entspräche dem in etwa der pretense awareness context. Dieser ist "a modification of the open one: both interactants are fully aware but pretend not to be." BARNEY GLASER/ANSELM STRAUSS, Awareness Contexts in Social Interaction, in: American Sociological Review 29 (1964), 669 ff., 670. 
recht, was los ist. ${ }^{34}$ In Zweifelsfällen wie in Fällen gescheiterter Ironisierungen sehen sich Sprecher gelegentlich sogar dazu veranlasst, ihre Äußerung im Nachhinein als ironisch zu charakterisieren (z. B. „das war ironisch gemeint"), oder aber auf die ernsten Implikationen hinzuweisen, die sie damit hatten eigentlich ansprechen wollen.

\section{Reaktionen der Ironisierten}

Wir haben schon darauf hingewiesen, dass die Mitarbeit der Rezipienten für das Gelingen ironischer Äußerungen wesentlich ist. Paradoxerweise gilt dies gerade auch für die eigentlichen Opfer ironischer Attacken. Sie beteiligen sich an ihrer eigenen Ironisierung indem sie auf ironische Attacken mit Redezugvakanzen oder ,ernst gemeinten' Rechtfertigungen reagieren. Aber auch das Ironiepublikum trägt seinen Teil bei. Der folgende Ausschnitt zeigt ein Beispiel für diese eigenartige Kooperation. Die Mitarbeit des Ironieobjekts besteht in diesem Fall darin, dass es nicht reagiert. Die entstehende Pause kann sich sehr lange hinziehen. Nicht nur die Nicht-Äußerung des Zeugen trägt in diesem Fall zur interaktiven Wirkung der Äußerung des Verteidigers bei, sondern vor allem der Umstand, dass dieser darauf verzichtet, die Reaktion des Zeugen einzufordern. In einem solchen Fall wäre als nächstes eigentlich der Richter als Verfahrenswalter gefordert die Verhandlung wieder an sich zu ziehen. Dieser aber zeigt kooperatives Schweigen, das er erst nach mehr als 20 Sekunden bricht, und zwar indem er ein neues Thema einführt. Man kann Pausen in derartigen Konstellationen als interaktive Strategien ansehen, die eingesetzt werden, um für alle Beteiligten erkennbar auf das Fehlen einer angemessenen Reaktion und damit indirekt auf den problematischen Status des Befragten hinzuweisen.

$\mathrm{V} \quad$ heut sagen sie klipp und klar

keinesfalls der Türke.

(0.2)

jetzt könn mer uns was aussuchen.

$\rightarrow \quad(4.6)$

?? ${ }^{\circ}(\mathrm{mh})^{\circ}$

$\rightarrow(9)$

?? ((Atmen, Geräusche))

$\rightarrow(7)$

${ }^{34}$ Nach Umberto Eco besteht das Schöne (und die Gefahr) an der Ironie darin, dass es immer jemanden gibt, der das ironisch Gesagte ernst nimmt; vgl. UMBERTO ECO, Nachschrift zum „Namen der Rose“. München 1984. 
ham Sie bei der Polizei gesagt es sei dieselbe Person

gewesen?

Verfahren Krapp, Zeugenaussage Werner, 06krap1a, 107/108

Eine weitere Variante möglicher Reaktionen von Ironisierten zeigt das nächste Beispiel. Hier wird der ironische Charakter der Episode ebenso wie die für die ironisierte Person problematischen Implikationen der ironischen Äußerung durch eine längere Schweigephase sowie durch die daran anschließende Reaktion des Zeugen deutlich gemacht. Indem dieser an die Pause eine Rechtfertigung anschließt, akzeptiert und relativiert er (durch die Gegenfrage) den in der ironisierenden Äußerung verkörperten Vorwurf.

$\mathrm{R}$ dafür gibt s keine andere Erklärung als dass sie ihm das:

(1.6)

auf sein(e)

(1.0)

seine nette Art hin

(0.4)

zur Verfügung stellen.

(3.0)

$Z \rightarrow{ }^{\circ}(. h h)^{\circ}$ e:hff:: ja. w wo hätt er s Geld sonst herkriegen;

Verfahren Krapp, Zeugenaussage Werner, 06-krap1a, 25/26

Im folgenden Ausschnitt schließlich reagiert der Angeklagte auf die Ironie zwar empört, aber durchaus ernsthaft. Seine Reaktion macht deutlich, dass er die ironische Absicht erkannt hat. Dies will er wohl zunächst thematisieren (,wollen Sie damit sagen ..."). Dann unterbricht er sich jedoch und begründet, warum die Post nicht ankam. Eine solche Begründung wäre redundant, wenn er die Äußerung des Richters als ernst gemeint verstünde.

$\mathrm{R} \quad{ }^{\circ}$ und (n Gesuch vom Land- von der

Amtsanwaltschaft Flaustadt is auch noch drin. ${ }^{\circ}$

(2.1)

A Weiß ich nich mehr.

$\mathrm{R} \quad{ }^{\circ}$ Wissen se überhaupt nischt ${ }^{\circ \circ}$

A Was soll des überhaupt ich weiß ${ }^{\circ \circ}$ ich kenn doch meine Akte nicht.

$\mathrm{R} \quad{ }^{\circ} \mathrm{ja}$. Deswegen erzähl ichs Ihnen ja was alles drin steht. ${ }^{\circ}$ 
A ()

(1)

$\mathrm{R}{ }^{\circ}$ (sch) wahrscheinlich auch die Post nich angekommen ${ }^{\circ \circ}$

(2.3)

A $\rightarrow$ wollen Sie damit sage wenn i i im Knast hock

is klar das die Post nich ankommt

Verfahren Jossen, Einlassung des Angeklagten 02-joss3.txt, 63

Nur geglückte Ironisierungsversuche eignen sich als Abschluss einer themenbezogenen Bezweiflungsphase. ${ }^{35}$ Ironische Attacken gelingen aber keineswegs in jedem Fall. Sie können insbesondere dann ins Leere laufen, wenn das Ironiesubjekt in seinem Redezug dem Angesprochenen mehrere Optionen der Reaktion offen lässt und dieser von daher seine Mitarbeit an der eigenen Ironisierung ohne unkooperativ zu erscheinen vermeiden kann. Im nachfolgenden Abschnitt bezieht sich der Zeuge in seiner Reaktion nicht auf die unmittelbar vorhergehende ironisierende Äußerung, sondern auf eine andere Frage, die der Staatsanwalt in seinem Redezug an früherer Stelle gestellt hatte. Er übergeht dann - ohne größere Pause - den Ironisierungsversuch und nimmt ihm damit viel von seiner Wirkung. Die Bezweiflungsphase kann an dieser Stelle nicht abgeschlossen werden. Stattdessen erfolgen weitere Fragen.

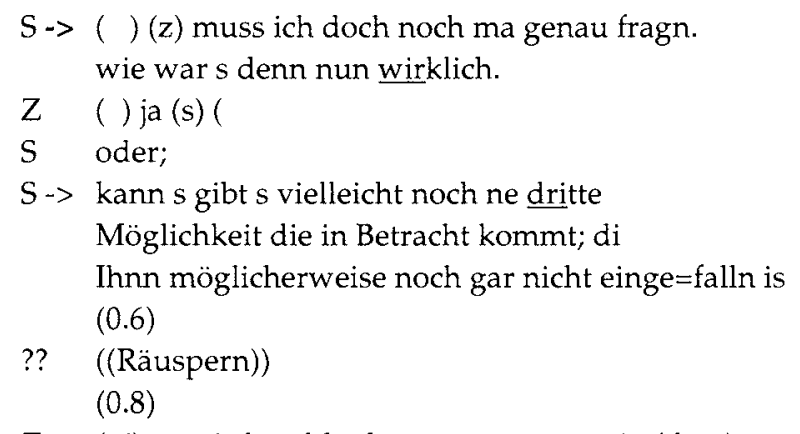

$\mathrm{Z}$-> (nj)a s wird wohl schon so gewesrn sein (dass)

( ) (s) Auto aus der Einfahrt rausfahrn wollte damith halt

Verfahren Krapp, Zeugenaussage Tupalla 20-brack5.txt, 25/26

Der „Bewusstheitskontext der Verstellung“, der für ironische Episoden während der Vernehmung kennzeichnend zu sein scheint, bezieht, so

\footnotetext{
35 Einschränkend muss man darauf hinweisen, dass sich empirisch keine ganz eindeutige Unterscheidung zwischen geglückt und nicht geglückt treffen lässt.
} 
können wir zusammenfassen, das Ironie-Publikum mit ein. Es entsteht eine stillschweigende Komplizenschaft zwischen dem Ironisierer und den Zuhörern. Typisch für diese Komplizenschaft ist, dass sie sich nicht oder nur ansatzweise offen äußert. ${ }^{36}$ Diese Komplizenschaft ist weder ganz verlässlich (aus der Sicht des Ironisierenden, der sich nie ganz der Wirkung seiner Ironie sicher sein kann), noch wirklich einklagbar (aus der Sicht aller Beteiligten) - etwa im Hinblick auf die Wertung der betreffenden Aussage in der Urteilsbegründung.

Insgesamt fällt allerdings auf, dass ironische Äußerungen durch ihre vielfältigen Markierungen und interaktiven Bestätigungen eine gewisse Prägnanz gewinnen. Zumindest scheinen sich die Details ironisch markierter Episoden den Beteiligten in besonderem Maße einzuprägen. Dies zeigt sich zum Beispiel darin, dass manche der Äußerungen, die Anlass zu ironischen Kommentaren gaben, in den Plädoyers und in den mündlichen Urteilsbegründungen wieder auftauchen - auch wenn ihre implizite Botschaft in der Situation selbst nicht formuliert worden war. In dem Prozess, aus dem der gerade zitierte Abschnitt stammt, verweisen sowohl der Staatsanwalt in seinem Plädoyer, wie anknüpfend daran die Richterin in ihrer mündlichen Urteilsbegründung auf die ironisch markierte Stelle und begründen damit ihre Feststellungen über die zweifelhafte Glaubwürdigkeit dieses Zeugen.

Aufschlussreich für die kommunikative Funktion der Ironie ist der Umstand, dass sich in den schriftlichen Urteilsgründen demgegenüber in keinem Fall ${ }^{37}$ ein Eingehen auf ironische Passagen während des Prozessverlaufs findet. Dies verweist wiederum auf die Situationsabhängigkeit der Ironie, auf ihre primär interaktive Wirkungsweise, sowie darauf, dass mündliche und schriftliche Urteilsbegründungen an unterschiedliche Adressaten gerichtet sind. Die Ironie entfaltet ihre Wirkung nur in der Situation, in der sie aufblitzt, und nur für jene, die daran beteiligt sind. Es wäre ein sehr ernstes Unterfangen mit kaum kalkulierbaren Konsequenzen in den Berufungsinstanzen, wollten die Richter versuchen in den schriftlichen Urteilsgründen über Ironie $z u$ berichten oder dort gar selbst ironisch zu werden.

\footnotetext{
${ }^{36}$ Einschränkend ist $z \mathfrak{u}$ bedenken, dass wir bei unseren Analysen auf Tonbandaufnahmen bzw. deren Transkriptionen angewiesen sind. In Beobachtungen haben wir viel sagende Blicke, Lächeln und ähnliche ,versteckte' Reaktionen bei den Ironisierern wie beim Ironiepublikum feststellen können. Allerdings erfolgen diese nicht einheitlich und sind auch nicht immer gegeben. Für eine strukturelle Analyse der Ironie im Verfahren würden derartige Hinweise auf Ironiesignale deshalb vermutlich selbst dann nicht ausreichen, wenn wir auf Videomaterial zurückgreifen können.

${ }^{37}$ Unser Korpus umfasst etwa 200 schriftliche Urteilsbegründungen
} 


\section{Ironie und Vorhalt}

Ironische Episoden zeigen auffällige Gemeinsamkeiten mit Vorhalten. Vorhalte werden insbesondere dazu eingesetzt, um Zeugen oder Angeklagte mit Widersprüchen zwischen ihren Aussagen in der Hauptverhandlung und anderen Beweismitteln, z. B. dem Protokoll der polizeilichen Vernehmung, zu konfrontieren. Vorhalte etablieren ähnlich wie ironische Äußerungen ein "mundanes Rätsel ${ }^{\prime 38}$, welches den befragten Personen zur Lösung vorgelegt wird. Vorhalte sind somit eindeutig Prüfoperationen. Vorhalte wie ironische Äußerungen gehen von der für das Alltagswissen selbstverständlichen Unterstellung aus, dass es nur eine wirkliche Version der in Frage stehenden Abläufe geben kann. Von daher sehen sich die betreffenden Zeugen und Angeklagten mit der moralischen - und eben nicht bloß kognitiven - Aufgabe konfrontiert das ihnen vorgehaltene „Rätsel“ aufzulösen.

Die Kunst des Vorhalts besteht darin, die vorgehaltenen Aussagen, Informationen oder Befunde so geschickt und so überzeugend miteinander zu kontrastieren, dass sie sich nicht nur gegenseitig ausschließen, sondern dass dadurch der damit konfrontierten Person die Möglichkeit genommen wird, sich auf die Subjektivität ihrer Wahrnehmungen bzw. Meinungen zurückzuziehen und das offerierte Rätsel als bloße Ansichtsache abzutun. Ironische Äußerungen bedienen sich der gleichen Kontrasttechnik wie Vorhalte. Von daher ist es nicht verwunderlich, wenn wir in der Umgebung ironischer Äußerungen gehäuft auf Vorhalte stoßen. Eine solche Verknüpfung von Vorhalt und Ironie zeigt der folgende Ausschnitt.

$\mathrm{Z}$.hh hhh ich meine mich erinnern zu können: einen Geldschein zusammen::zudrehn: dass er also nen Geldschein zusammengedreht hat un:d hath damith e auf: aufgeschnieft oder in die Nase gezogen (,) geschnieft

$\mathrm{V}->$ jaja gut e: damals war s nur zur Klarstellung damals hatte er es so ham sie s bei der Polizei gesagt so hat $\mathrm{s}$ die Polizei aufgeschrieben .hh damals hatte er es noch (.) geraucht. (0.4)

$\mathrm{V} \quad$ ich versuch ja nur irgendwie $-\mathrm{h}$ ich versuch nur ne Version zu finden

\footnotetext{
38 Melvin POllner, Mundanes Denken, in: Elmar Weingarten/Fritz SACK/JiM
} SCHENKEIN (Hrsg.), Ethnomethodologie. Frankfurt am Main 1976, 295-326. 
$\mathrm{Z} \quad=\mathrm{ja}$

$\mathrm{V} \quad=\mathrm{e}$ : nachdem sie uns verschiene bieten, (0.2) versuch ich $\mathrm{n}$ Weg zu finden was eigntlich hier richtich

sein kann.

Verfahren Krapp, Zeugenaussage Werner 06-krap1a.txt, 132/133

Der Verteidiger hält dem Zeugen seine Aussage bei der Polizei vor. Ausweislich des Protokolls hatte dieser gesagt, der Angeklagte habe das Rauschgift geraucht. Nimmt man die Äußerung, "damals hatte er es noch (...) geraucht", wörtlich, so könnte man schließen, das Ereignis habe sich mittlerweile verändert. Möglich ist dies jedoch nur bei einer fiktiven Geschichte. Die Schilderung des Zeugen wird wie ein Roman oder ein Märchen behandelt, nicht aber als ernst gemeinte Darstellung der Wirklichkeit. Ironisch (besser: potentiell ironisch) ist dann die folgende Äußerung des Verteidigers, der Zeuge biete dem Gericht verschiedene Versionen an, und er, der Verteidiger versuche "ja nur irgendwie" eine Version zu finden, die richtig sein kann.

Durch dieses Hintereinanderschalten von Vorhalt und Ironie wird die Bezweiflung nicht allein wiederholt, sondern verstärkt. Ironien sind somit zum einen mit Vorhalten strukturell verwandt (beide enthalten Kontraststrukturen); zum anderen funktional äquivalent (im Hinblick auf den Ausdruck von Zweifel); und schließlich lassen sie sich so kombinieren, dass dadurch Steigerungseffekte der Infragestellung entstehen. Für Letzteres spricht nicht nur der Umstand, dass Ironien in allen Fällen nach den Vorhalten in eine Bezweiflungssequenz eingebaut werden, sondern ebenso, dass nach geglückten Ironien in der Regel ein mehr oder weniger ausgeprägter Wechsel des thematischen Angriffspunktes und/oder ein Wechsel der befragenden Person folgt. Waren es Staatsanwälte und Verteidiger, die die Ironie platzierten, so nimmt meist der Richter von sich aus wieder die Befragung in die Hand, wie im folgenden Ausschnitt:

((Der Zeuge hatte nach dem polizeilichen Vernehmungsprotokoll ausgeführt, er habe sich mit einem bestimmten Türken in einer Gaststätte getroffen. In dieser Zeugenvernehmung dagegen spricht er davon, er habe sich nicht mit ,dem Türken', sondern mit einer anderen Person getroffen. Darauf spielt der Verteidiger mit seiner Äußerung, jetzt könn mer uns was aussuchen' an.))

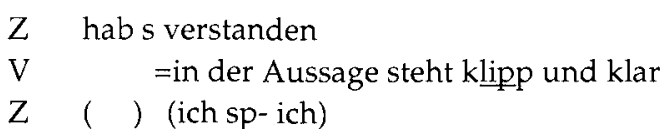


V drin es war der -

Z spreche doch von zwei verschiedenen Personen.

$\mathrm{V} \quad$ =s war klipp

$\mathrm{V}$ un klar der Türke heut sagen sie klipp und klar keinesfalls der Türke.

$(0.2)$

$\rightarrow \quad$ jetzt könn mer uns was aussuchen.

?? ${ }^{\circ}(\mathrm{mh})^{\circ}$

(9)

?? ((Atmen, Geräusche))

(7)

R $\rightarrow$ ham Sie bei der Polizei gesagt es sei dieselbe Person gewesen?

Verfahren Krapp, Zeugenaussage Werner, 06krapla, 107/108

Der Verteidiger beschränkt sich nicht auf den Vorhalt im engeren Sinne. Er schließt daran - ohne eine Stellungnahme des Zeugen zu dem offerierten Rätsel abzuwarten - gleich noch eine ironische Interpretation der durch das Verhalten des Zeugen entstandenen Situation an (,jjetzt könn mer uns was aussuchen"). Im Endeffekt hat er damit zwei Äußerungen produziert, zu denen jeweils eine Stellungnahme erwartet werden kann. Aufgrund der für unseres Sprechaustauschsystem typischen Präferenz für direkte Anschlüsse (,contiguity') wäre jetzt zunächst einmal eine Stellungnahme zur letzten Äußerungseinheit (der Feststellung über die Beliebigkeit der Auswahl) zu erwarten. Damit ist der Vorhalt begründende Widerspruch nur mehr indirekt, und wenn überhaupt, dann nur noch mit erhöhtem kommunikativem Aufwand in der nächsten Äußerung ansprechbar. Implizit wird der Vorhalt als schon nicht mehr behebbar bzw. nicht mehr einer Klärung bedürftig behandelt. Das macht die verhältnismäßig lange Redezugvakanz verständlich, die auf die Äußerung des Verteidigers ,,jetzt können wir uns was aussuchen" eintritt. Da auch der Verteidiger seine Vernehmung des Zeugen nicht fortsetzt, entsteht eine Pause, während der der Vorhalt und seine ironische Kommentierung quasi im Raume stehen. Erst nach fast 25 Sekunden greift schließlich der Richter mit einer Nachfrage ein.

Beim Vorhalt kann es offen bleiben, ob es für den festgestellten Widerspruch noch andere Erklärungen gibt als die, dass der Zeuge bewusst die Unwahrheit sagte. Der ironische Vorhalt dagegen repräsentiert eine zwar nicht ausgesprochene, aber offensichtlichere, sozusagen eine sozial endgültigere Bezweifelung des Wahrheitsgehalts einer Aussage. Die Ironie steht nicht nur mit dem Vorhalt in einem systemati- 
schen und sequentiellen Zusammenhang, sondern hat auch ihren festen interaktionslogischen Ort in der Dramaturgie der Befragung von Zeugen und Angeklagten. Praktisch alle Ironisierungen sind so platziert. Sie fungieren somit als Schluss- bzw. Höhepunkte von Bezweiflungsprozessen.

\section{Funktionen von Ironie: Schaffung von Gemeinschaft und Differenz}

Manche Theoretiker des Humors vermuten, die interaktive Funktion ironischer Bemerkungen bestünde primär in der Ausübung sozialer Kontrolle. ${ }^{39}$ Insofern ,soziale Kontrolle' jene Prozesse und Mechanismen bezeichnen soll, die eingesetzt werden, um Gesellschaftsmitglieder zu bestimmten, als positiv oder erwünscht angesehenen Verhaltensweisen $\mathrm{zu}$ bewegen, bieten unsere Daten dafür keine Belege. Auf ironische Bemerkungen geschieht typischerweise nichts. Meist folgt ein Schweigen und/oder das Ironieobjekt versucht (dann) eine möglichst ernsthafte Bestätigung seiner bisherigen Aussage. In keinem Fall haben wir eine Selbstkorrektur der Ironisierten beobachtet, was bei einem funktionierenden sozialen Kontrollmechanismus zu erwarten gewesen wäre.

Es wäre ebenso irreführend, in der Ironie nur den Ausdruck bzw. die demonstrative Inszenierung einer Machtasymmetrie zwischen den Verfahrensbeteiligten erblicken zu wollen. Zumindest wäre es eine recht riskante Strategie in Gerichtsverhandlungen Ironie dazu einzusetzen. Ironisierungen machen ja gerade den Ironisierer für Angriffe verletzlicher. Auf jeden Fall gilt diese Feststellung für Richter, die sich dieser Gefahr gemeinhin wohl bewusst sind und deshalb in den entsprechenden Fällen besondere Vorsicht walten lassen, um sich nicht der Gefahr eines Befangenheitsantrags auszusetzen.

Zutreffender wäre davon sprechen, dass durch Ironie im Strafverfahren Differenz etabliert wird zwischen denen, die über die Ironie offen, häufiger aber insgeheim schmunzeln (also die bzw. ein Teil der Zuhörer) und jener Person, die gerade ironisiert wird. Die Ironie übt ihre Wirkung in doppelter Weise aus: sie schafft eine mehr oder weniger heimliche Gemeinschaft derjenigen, welche die implizite Botschaft vor dem Hintergrund der Verfahrensgeschichte verstehen (dazu gehört oft auch das Ironieobjekt). Zugleich konstituiert sie eine Differenz zwi-

\footnotetext{
$39 \mathrm{Vgl}$. als repräsentativen Überblick über einschlägige Positionen MichaEL MULKAY, On Humor. Its Nature and its Place in Modern Society. Cambridge 1988.
} 
schen der ironisierten Person und allen anderen. Die Ironie spielt immer auf ein gemeinsames Hintergrundwissen an (dazu gehört insbesondere die vorherige Befragungs- bzw. Bezweiflungssequenz, aber auch der bisherige Prozessverlauf), dessen Inrechnungstellen für eine adäquate, d. h. auf die Frage der Glaubwürdigkeit bezogene Interpretation der Intention der Ironie notwendig ist. Auf diese gemeinsame Interaktionsgeschichte spielt die Ironie nicht nur an, sie liefert darüber hinaus deren dramatischen Höhepunkt. Sie tut dies in einer besonders markierten Weise, wodurch nicht nur die betreffende Episode im Gruppengedächtnis konserviert, sondern auch der gemeinsame Erfahrungsbestand bestätigt und bekräftigt wird.

Die Ironie fungiert somit als Mechanismus der Irritation und der Vergemeinschaftung gleichermaßen. Der Aspekt des Humorigen spielt dabei keineswegs die entscheidende Rolle. ${ }^{40}$ Allerdings kann er die Vergemeinschaftungswirkung der Ironie positiv unterstützen. Schon Schleiermacher hat von der Ironie als einem Hilfsmittel gesprochen, „in der größeren Gesellschaft eine kleinere geheime zu errichten". Wenn sehr unterschiedliche Menschen zu einer "Gesellschaft" (sprich: einem mündlichen Verfahren) zusammen gezwungen seien

,.... so giebt es ja kein höflicheres und freundlicheres Hülfsmittel, als, die Einheit bestehen zu lassen, und zur Herzenserleichterung neben ihr und in ihr, ohne Nachtheil der Anderen, insgeheim, wenn gleich nur auf vorübergehende Augenblicke, eine reizendere und gehaltvolle Vereinigung zu stiften. ${ }^{\mu 1}$

Nachdem wir die kommunikative Methodik der Ironie bestimmt haben, bleibt als Letztes die Frage zu beantworten, was die besondere interaktive Leistung der Ironie für das Gerichtsverfahren, genauer, im Kontext der Vernehmung von Angeklagten und Zeugen ausmacht.

Wir hatten schon oben auf den Umstand aufmerksam gemacht, dass die am Verfahren beteiligten Juristen, vor allem aber die Richter es vermeiden, eindeutige Einschätzungen zur Beweislage noch während der Beweiserhebung bekannt zu geben. Gleichwohl besteht zwischen ihnen hinsichtlich der Würdigung von Zeugen und ihren Aussagen zumin-

\footnotetext{
${ }^{40}$ Nach WEINRICH, Ironie (Fn. 15), gerät die Ironie überhaupt erst im 19. Jahrhundert in eine Begriffsnachbarschaft zum Humor, wobei das Verhältnis beider Begriffe bis heute in einer gewissen Ambivalenz verbleibt. Dazu ist wohl die Ironie eine zu ernste Angelegenheit. Dies scheint Jean Paul im Blick gehabt zu haben, wenn der der Ironie im Unterschied zum Humor die Qualitäten des Kalten und Bitteren zuweist und vom „Ernst der Ironie" spricht.

41 FRIEDRICH D. E. SCHLEIERMACHER, Versuch einer Theorie des geselligen Betragens, in: Kritische Gesamtausgabe, Erste Abteilung: Schriften und Entwürfe. Bd 2. Herausgeben von Gerhard EBeling/Hermann FisCHER/Heinz KimMerle. Berlin 1984, 165 ff., 183.
} 
dest in den meisten Fällen eine weitgehende, wenn auch stillschweigende Übereinstimmung. Unsere Untersuchung hat eine Reihe von Belegen für die These erbracht, dass bei diesem mehr oder weniger beiläufigen Verständigungsprozess die kommunikative Modalität der Ironie eine bedeutsame Rolle spielt, und dass die Wirkung der Ironie mit der besonderen Methodizität dieses Äußerungsformates zusammenhängt. Ironisierungen können als ein Instrument eingesetzt werden, um für alle praktischen Zwecke Glaubwürdigkeit bzw. Unglaubwürdigkeit in der Situation zu etablieren - ohne dabei die interaktiven und strategischen Nachteile eindeutiger Festlegungen in Kauf nehmen zu müssen. Sie erlauben es (sofern sie nicht von einem der Beteiligten, z. B. von einem Verteidiger, zurückgewiesen werden) ein, vorläufiges Zwischenergebnis einzufahren' und in der laufenden Verhandlung ,Meilensteine zu setzen', und zwar in einer für alle sichtbaren und später rekapitulierbaren Weise.

In Zusammenfassung unserer analytischen Rekonstruktion der sozialen Organisation von Ironie lassen sich die folgenden acht Aspekte nennen, welche Ironie für die Zwecke der Verständigung des Gerichts über Unglaubwürdigkeit hilfreich erscheinen lassen:

1. Ironisierungen erlauben es den Ironieobjekten wie den anderen Gerichtsbeteiligten gegenüber Zweifel deutlich zu machen, ohne dass dagegen von Seiten des Ironisierten mit großer Aussicht auf Erfolg interveniert werden könnte.

2. Mit ihrer Hilfe sind Bezweiflungen möglich, ohne dass der Zweifel ausdrücklich als solcher markiert und ggf. verteidigt werden müsste.

3. Sie gestatten es Aufmerksamkeit zu fokussieren und Hinweise zu geben, ohne gleich zu endgültigen und gleichsam offiziellen Festlegungen greifen zu müssen.

4. Sie ermöglichen, Bezweiflungsphasen abzuschließen und zu neuen Themen überzugehen. Angesichts der Ausrichtung der Gerichtskommunikation darauf, es erst ganz zum Ende des Verfahrens zu endgültigen Entscheidungen kommen $z u$ lassen, gibt es keine immanente Stoppregel für die Befragungs- und Bezweiflungsaktivitäten. Hier kann die Ironie, insoweit es auf sie von Seiten der Ironieobjekte wie des Publikums nichts mehr zu sagen gibt, den Gordischen Knoten einer Kommunikation, die sich im Kreise dreht, lösen helfen. 
5. Mit Ironisierungen lassen sich erhebliche interaktive Wirkungen erzielen, ohne dass die verschiedenen Beteiligten ihr Gesicht verlieren müssten. Das Objekt einer erfolgreichen Ironie ist zwar sozial angeschlagen, nicht aber im Garfinkelschen Sinn degradiert.

6. Die Ironisierenden und das Publikum gehen dabei nicht das Risiko ein, auf bestimmte Feststellungen und Absichten vorschnell festlegbar zu sein. Aufgrund ihrer gekonnten Intransparenz ermöglicht es die Ironie, die Untiefen der Moral zu vermeiden und dennoch Stellung zu beziehen. ${ }^{42}$ Man könnte im Zusammenhang der Verwendung von Ironie im Verfahren auch von taktvoller Entschiedenheit sprechen. ${ }^{43}$

7. Ironisierungen gestatten es, ein Verfahren in relativ ruhigen Bahnen ablaufen zu lassen und gleichwohl erheblichen Dissens zwischen den Beteiligten zum Ausdruck zu bringen. Ironisierungen dienen somit nicht nur der gemeinsamen "Wahrheitsfindung"; sie ermöglichen darüber hinaus, die Ernsthaftigkeit des Verfahrens aufrechtzuerhalten.

8. Die interaktiv geglückte, oder wie Schlegel formulierte, „die vollendete absolute Ironie" hört auf Ironie zu sein und wird ernsthaft. Sie durchbricht freilich nicht den Ernst der Verfahrenskommunikation, wie man vermuten könnte, sie ermöglicht ihn, allerdings auf einer neuen Stufe, nämlich der eines Ernstes zweiter Ordnung:

„Der Ernst erster Ordnung meint, was er sagt, und sucht die Bedingung dieses Meinens in der Sache, um die es geht. Sein Ernst ist der Ernst der Verhältnisse, der Ernst der Welt. Der Ernst zweiter Ordnung lässt offen, ob er meint, was er sagt, und verweist nur darauf, dass er etwas sagt und sicherlich auch etwas meint. Er lädt ein zur Kommunikation ... Der Ernst zweiter Ordnung ist immer nur der Ernst des anderen, der mich ernst nimmt beziehungsweise die Situation, die Kommunikation, die Verhältnisse. Dieser Ernst ist auch ein Ernst der Verhältnisse, aber nicht ein Ernst der Welt, sondern nur der Kommunikation in und über die Welt." ${ }^{44}$

\footnotetext{
42 NikLaS LuHManN, Die Gesellschaft der Gesellschaft. Frankfurt am Main 1997, 1039.

${ }^{43}$ Erving Goffman diskutiert ähnliche Phänomene unter dem Überbegriff der ",andeutenden Kommurikation"; siehe ERvING GOFFMANN, Das Individuum im öffentlichen Austausch. Frankfurt am Main 1974, $36 \mathrm{f}$.

${ }^{44}$ BAECKER, Ernste Kommunikation (Fn. 4), 389.
} 


\section{Literatur}

Baecker, Dirk, Ernste Kommunikation, in: Karl Heinz Bohrer (Hrsg.), Sprachen der Ironie - Sprachen des Ernstes, Frankfurt am Main 2000, 389-403.

Bender Rolf/Nack, Armin, Tatsachenfeststellung vor Gericht. 2 Bände. München 1995.

Berg, Wolfgang, Uneigentliches Sprechen. Zur Pragmatik und Semantik von Metapher, Metonymie, Ironie und rhetorischer Frage. Tübingen 1978.

Bergmann, Jörg R., Schweigephasen im Gespräch, in: Hans-Georg Soeffner (Hrsg.), Beiträge zu einer empirischen Sprachsoziologie. Tübingen 1982, 143184.

Bollnow, Otto F., Ironie, in: Ders., Die Ehrfurcht. 2. Aufl. Frankfurt am Main 1958 (Originalausgabe 1947), 147-179.

Brown, Penelope, Levinson, Stephen, Politeness. Some Universals in Language Usage. Cambridge 1987.

Eco, Umberto, Nachschrift zum "Namen der Rose". München, Wien 1984.

Glaser, Barney/Strauss, Anselm, Awareness Contexts in Social Interaction. American Sociological Review 29 (1964), 669-679.

Goffman, Erving, Das Individuum im öffentlichen Austausch. Frankfurt am Main 1974.

Heritage, John, Garfinkel and Ethnomethodology. Cambridge 1984.

Japp, Uwe, Theorie der Ironie. Frankfurt am Main 1983.

Kotthoff, Helga, Ironie in Privatgesprächen und Fernsehdiskussionen, in: Inken Keim/Wilfried Schütte (Hrsg.), Soziale Welten und kommunikative Stile. Tübingen 2002, 445-473.

Lapp, Edgar, Linguistik der Ironie. Tübingen 1992.

Lewandowski, Theodor, Linguistisches Wörterbuch. 5. Aufl., Heidelberg 1990.

Luhmann, Niklas, Legitimation durch Verfahren. Neuwied 1969.

Luhmann, Niklas, Die Gesellschaft der Gesellschaft. Frankfurt am Main 1997.

Meier, Christoph, Arbeitsbesprechungen. Interaktionsstruktur, Interaktionsdynamik und Konsequenzen einer sozialen Form. Opladen 1997.

Mulkay, Michael, On Humor. Its Nature and its Place in Modern Society. Cambridge 1988.

Pollner, Melvin, Mundanes Denken, in: Elmar Weingarten/Fritz Sack/Jim Schenkein (Hrsg.), Ethnomethodologie. Frankfurt am Main 1976, 295-326.

Reinicke, Gerhard, Die Krise der Beweiswürdigung im Strafprozess oder Über die Schwierigkeit, einem Zeugen nicht zu glauben, in: Monatsschrift für Deutsches Recht 8 (1986), 630-637.

Sacks, Harvey, Lectures on Conversation. 2 Bände, Oxford 1992.

Schleiermacher, Friedrich D. E., Versuch einer Theorie des geselligen Betragens, in: Kritische Gesamtausgabe, Erste Abteilung: Schriften und Entwürfe, Bd. 2. 
Herausgegeben von Gerhard Ebeling, Hermann Fischer und Heinz Kimmerle. Berlin 1984 (Originalausgabe 1878), 165-184.

Weinrich, Harald, Ironie, in: Joachim Ritter/Karlfried Gründer (Hrsg.), Historisches Wörterbuch der Philosophie. Bd. 4. Darmstadt 1976, 577-582.

Wolff, Stephan, Text und Schuld. Die Rhetorik psychiatrischer Gerichtsgutachten. Berlin 1995.

Wolff, Stephan/Müller, Hermann, Ironie als Instrument der ,Wahrheitsfindung', in: Zeitschrift für Soziologie 24 (1995), 451-464.

Wolff, Stephan/Müller, Hermann, Kompetente Skepsis. Eine konversationsanlytische Untersuchung zur Glaubwürdigkeit in Strafverfahren. Opladen 1997. 